\title{
Distinct Laterality in Forelimb-Movement Representations of Rat Primary and Secondary Motor Cortical Neurons with Intratelencephalic and Pyramidal Tract Projections
}

\author{
(DShogo Soma, ${ }^{1,3,4}$ Akiko Saiki, ${ }^{1,3}$ Junichi Yoshida, ${ }^{1,4}$ Alain Ríos, ${ }^{2}$ Masanori Kawabata, ${ }^{2}$ Yutaka Sakai, ${ }^{1,2,3}$ \\ and Yoshikazu Isomura ${ }^{1,2,3}$ \\ ${ }^{1}$ Brain Science Institute and ${ }^{2}$ Graduate School of Brain Sciences, Tamagawa University, Tokyo 194-8610, Japan, ${ }^{3}$ Brain/MINDS and ${ }^{4}$ Japan Society for the \\ Promotion of Science, Tokyo 102-0083, Japan
}

Two distinct motor areas, the primary and secondary motor cortices (M1 and M2), play crucial roles in voluntary movement in rodents. The aim of this study was to characterize the laterality in motor cortical representations of right and left forelimb movements. To achieve this goal, we developed a novel behavioral task, the Right-Left Pedal task, in which a head-restrained male rat manipulates a right or left pedal with the corresponding forelimb. This task enabled us to monitor independent movements of both forelimbs with high spatiotemporal resolution. We observed phasic movement-related neuronal activity (Go-type) and tonic hold-related activity (Hold-type) in isolated unilateral movements. In both M1 and M2, Go-type neurons exhibited bias toward contralateral preference, whereas Hold-type neurons exhibited no bias. The contralateral bias was weaker in M2 than M1. Moreover, we differentiated between intratelencephalic (IT) and pyramidal tract (PT) neurons using optogenetically evoked spike collision in rats expressing channelrhodopsin-2. Even in identified PT and IT neurons, Hold-type neurons exhibited no lateral bias. Go-type PT neurons exhibited bias toward contralateral preference, whereas IT neurons exhibited no bias. Our findings suggest a different laterality of movement representations of M1 and M2, in each of which IT neurons are involved in cooperation of bilateral movements, whereas PT neurons control contralateral movements.

Key words: channelrhodopsin-2; collison test; contralateral/ipsilateral; cortical hierarchy; pyramidal cell; rat

Significance Statement

In rodents, the primary and secondary motor cortices (M1 and M2) are involved in voluntary movements via distinct projection neurons: intratelencephalic (IT) neurons and pyramidal tract (PT) neurons. However, it remains unclear whether the two motor cortices (M1 vs M2) and the two classes of projection neurons (IT vs PT) have different laterality of movement representations. We optogenetically identified these neurons and analyzed their functional activity using a novel behavioral task to monitor movements of the right and left forelimbs separately. We found that contralateral bias was reduced in M2 relative to M1, and in IT relative to PT neurons. Our findings suggest that the motor information processing that controls forelimb movement is coordinated by a distinct cell population.

\section{Introduction}

Several motor areas in primate cerebral cortex have hierarchies characterized by their own functions. The higher-order motor

Received May 1, 2017; revised Sept. 20, 2017; accepted Sept. 22, 2017.

Author contributions:S.S. and Y.I. designed research;S.S., A.S., J.Y., A.R., and M.K. performed research; S.S., A.S., Y.S., and Y.I. contributed unpublished reagents/analytic tools; S.S., A.S., and Y.S. analyzed data; S.S., A.R., Y.S., and Y.I. wrote the paper.

This work was supported by Grants-in-Aid for Japan Society for the Promotion of Science Research Fellow JP15J00807 to S.S. and JP16J11697 to J.Y., AMED Brain/MINDS to Y.I., Grants-in-Aid for Scientific Research on Innovative Areas JP16H01516 to Y.S. and JP26112005 and JP16H06276 to Y.I., and MEXT Supported Program for the Strategic Research Foundation at Private Universities to Y.S. and Y.I. We thank Drs. K. Enomoto, M. Kimura, K. Kobayashi, A. Nambu, S. Nonomura, T. Samura, K. Yamanaka, and H. Yawo for helpful suggestions and discussions; and M. Goto, Y. Maruyama, C. Soai, and H. Yoshimatsu for technical assistance.

The authors declare no competing financial interests. areas, including the premotor cortex (PM) and supplementary motor areas (SMA), are involved in processing abstract information regarding limb movements (Weinrich and Wise, 1982; Mushiake et al., 1991; Shima et al., 1996; Hoshi and Tanji, 2000; Churchland et al., 2006), whereas the primary motor cortex (M1) is involved in processing concrete motor information (Evarts, 1966; Tanji et al., 1987; Griffin et al., 2015). According to such

Correspondence should be addressed to Dr. Shogo Soma, Brain Science Institute, Tamagawa University, 6-1-1 Tamagawa-gakuen, Machida, Tokyo 194-8610, Japan. E-mail: s.soma@lab.tamagawa.ac.jp.

A. Saiki's present address: Department of Neurobiology, Institute of Biomedical and Health Sciences, Hiroshima University, Hiroshima 734-8553, Japan.

DOl:10.1523/JNEUROSCI.1188-17.2017

Copyright $\odot 2017$ the authors $\quad 0270-6474 / 17 / 3710904-13 \$ 15.00 / 0$ 
functional characteristics, the motor laterality (the neuronal selectivity for movements of the right and left limbs) depends on the hierarchical level. Neuronal activity in M1 and PM is predominantly associated with contralateral and bilateral arm movements, respectively (Kurata, 2010), and SMA neurons exhibit intermediate degrees of motor laterality between M1 and PM (Nakayama et al., 2015). Thus, the higher-order motor areas exhibit less laterality than M1 (Evarts, 1966; Tanji et al., 1987; Cisek et al., 2003; Kurata, 2007).

Rodents have two motor areas, M1 and secondary motor cortex (M2). Based on their axonal connectivity and motor response to intracortical microstimulation, $\mathrm{M} 1$ and $\mathrm{M} 2$ are thought to be homologous to the primate $\mathrm{M} 1$ and PM/SMA, respectively (Donoghue and Wise, 1982; Neafsey et al., 1986; Reep et al., 1987; Rouiller et al., 1993; Deffeyes et al., 2015; Hira et al., 2015). Rodents are becoming increasingly important as experimental animals for investigating motor information processing because they are the most suitable models for genetic and optical manipulation/identification of specific neurons and circuits (Li et al., 2015, 2016; Tantirigama et al., 2016; Saiki et al., 2017). Nevertheless, the differences in how M1 and M2 exhibit the laterality in forelimb-movement representations remain uncharacterized, largely because most rodent experiments to date have been performed under freely moving conditions (Kitsukawa et al., 2011; Soma et al., 2014; Kawai et al., 2015), which makes it extremely difficult to measure independent movements of both forelimbs. Recently, several studies using head-restrained rodents attempted to examine motor information processing of body parts, including the tongue (Li et al., 2015), whiskers (Ebbesen et al., 2017), and forelimbs (Isomura et al., 2009). However, none of these studies evaluated motor laterality because (1) the tongue is a single organ in the midline, and its related neuronal activity represents just the direction but not laterality; (2) the right and left whiskers always move synchronously (Carvell and Simons, 1990; Gao et al., 2001); and (3) no behavioral tasks were available that allowed separate monitoring of each forelimb. Therefore, to examine the motor laterality, it was necessary to develop a behavioral task capable of inducing independent forelimb movements.

The output layers of the motor areas have two classes of pyramidal cells with specific morphologies and axonal projections: the intratelencephalic (IT) and the pyramidal tract (PT) neurons (Shepherd, 2013; Harris and Shepherd, 2015). IT neurons send axonal projections bilaterally to other areas within the telencephalon. By contrast, PT neurons send projections to the ipsilateral cortex, striatum, thalamus, pontine nucleus, and contralateral spinal cord, but no bilateral projections (Reiner et al., 2003; Morishima and Kawaguchi, 2006). Therefore, it is possible that these projection neurons functionally represent differences in motor laterality. In this study, we developed a novel behavioral experiment system requiring right-left forelimb control in rodents and examined the differences in motor laterality between M1 and M2, as well as between antidromically identified IT and PT neurons, using an optogenetic spike collision method in transgenic rats. To the best of our knowledge, this is the first study that shows the laterality of cortical motor representation and its areal difference in rodents.

\section{Materials and Methods}

Animals and surgery. All experiments were approved by the Animal Research Ethics Committee of Tamagawa University (animal experiment protocol H22/27-32) and were performed in accordance with the Fundamental Guidelines for Proper Conduct of Animal Experiment and Related Activities in Academic Research Institutions (Ministry of Educa- tion, Culture, Sports, Science, and Technology of Japan) and the Guidelines for Animal Experimentation in Neuroscience (Japan Neuroscience Society). All surgical procedures were performed under appropriate isoflurane anesthesia (see below), and all efforts were made to minimize suffering. Our procedures for animal experiments were established in our previous studies (Isomura et al., 2009; Kimura et al., 2012; Nonomura et al., 2017).

Sixteen adult W-TChR2V4 rats ( $272 \pm 26 \mathrm{~g}$, males) that expressed the ChR2-Venus conjugate under the control of the Thy1.2 promoter (Tomita et al., 2009) were kept in their home cage under an inverted light schedule (lights off at 9:00 A.M.; lights on at 9:00 P.M.). These rats were briefly handled by the experimenter (10 min, twice) before the surgery. For head-plate (CFR-2, Narishige) implant, animals were anesthetized with isoflurane $(4.5 \%$ for induction and $2.0 \%-2.5 \%$ for maintenance, Pfizer) using an inhalation anesthesia apparatus (Univentor 400 anesthesia unit, Univentor) and placed on a stereotaxic frame (SR-10R-HT, Narishige). For local anesthesia, lidocaine (Astra Zeneca) was administered around the surgical incisions. The reference and ground electrodes (Teflon-coated silver wires, A-M Systems; $125 \mu \mathrm{m}$ in diameter) were implanted above the cerebellum. During anesthesia, body temperature was maintained at $37^{\circ} \mathrm{C}$ using an animal warmer (BWT-100, Bio Research Center). Analgesics and antibiotics were applied postoperatively as required (meloxicam, $1 \mathrm{mg} / \mathrm{kg}$ s.c., Boehringer Ingelheim; gentamicin ointment, $0.1 \%$ ad usum externum, MSD). In some experiments ( $n=6$ rats), a twisted Teflon-coated silver wire electrode was implanted into the left upper forelimb (near the biceps brachii muscle) to measure EMG activity.

After full recovery from surgery ( $6 \mathrm{~d}$ later), rats had ad libitum access to water during weekends, but during the rest of the week obtained water only by performing the task correctly. When necessary, an agar block (containing $15 \mathrm{ml}$ water) was given to the rats in their home cage to maintain them at $>85 \%$ of original body weight (Saiki et al., 2014).

Behavioral task. We developed the Right-Left Pedal task in our operant conditioning system (custom-made by O'Hara; see Fig. $1 A$ ) to examine the selectivity of contralateral or ipsilateral forelimb movement in the neuronal activity of the motor cortices (M1 and M2) of rats. In this task, the rats had to manipulate the right and left pedals with the corresponding forelimb in a head-fixed condition. They spontaneously started each trial by pushing both pedals down with the right and left forelimbs and holding them for a short period ("holding period," at least $1 \mathrm{~s}$; within a holding area, $0 \%-30 \%$ in relative pedal position). After the holding period was completed, they had to choose either the right or left pedal without any instruction cue, and then release it to obtain $0.1 \%$ saccharin water $(10 \mu \mathrm{l})$ as a reward. This task consisted of two blocks, right pedalrewarded and left pedal-rewarded, and the rats had to choose the appropriate pedal depending on the context. Each block lasted until the rat performed $>30$ correct trials and achieved correct performance of $80 \%$ in the most recent 10 trials. The reward was dispensed from the tip of a spout by a micropump with a $300-700$ ms delay (100 ms steps at random). The reward delivery period was followed by a short intertrial interval $(1 \mathrm{~s})$. If the rats chose the incorrect pedal, they were not rewarded (error trial), and only an error sound was given $(3 \mathrm{kHz}, 300 \mathrm{~ms})$. If they did not complete the holding period (immature trial), no feedback was given (no reward and no error sound). The rats typically learned this task within 2 weeks ( $2-3$ h a day; see Fig. $1 B$ ).

Once the rats completed the operant learning of the Right-Left Pedal task, they underwent a second surgery under anesthesia, and tiny holes $(1.0-1.5 \mathrm{~mm}$ in diameter) were made in the skull and dura mater above M1 (1.0 mm anterior, $\pm 2.5 \mathrm{~mm}$ lateral from bregma) and M2 (3.5 mm anterior, $\pm 2.4 \mathrm{~mm}$ lateral from bregma) (Saiki et al., 2017). These coordinates were determined by intracortical microstimulation $(50-100 \mu \mathrm{A}$, 50 pulses at $100 \mathrm{~Hz}$ ) to evoke reliable movements of the contralateral forelimb in our preliminary experiments (Saiki et al., 2014, 2017; Kimura et al., 2017; data not shown). In addition, these coordinates were confirmed to evoke reliable movements of the contralateral forelimb by optogenetic stimulation with blue light-emitting diode (LED) light pulses $(460 \mathrm{~nm}, 5-10 \mathrm{~mW}, 20$ pulses at $100 \mathrm{~Hz})$ after recordings. To perform the optogenetic light-induced spike collision (see below), additional tiny holes were made above the ventral nuclei of the thalamus (4.0 $\mathrm{mm}$ posterior, $\pm 2.0-3.5 \mathrm{~mm}$ lateral from bregma) and pontine 
nuclei (7.0 mm posterior, $\pm 1.0 \mathrm{~mm}$ lateral from bregma). All holes were immediately covered with silicon sealant (DentSilicone-V, Shofu).

Electrophysiological recording. We performed extracellular multineuronal (multiple isolated single-unit) recordings from individual neurons in the output layer (layer 5) of motor cortices (Isomura et al., 2009; Saiki et al., 2017) while the rats were performing the behavioral task. Supported by agarose gel ( $2 \%$ agarose-HGT, Nacalai Tesque) on the brain, 32-channel silicon probes (a32-Isomura-6-14-r2-A32 or ISO-3x-tetA32; NeuroNexus Technologies) (Saiki et al., 2017) were precisely inserted into M1 and M2, up to $1250 \mu \mathrm{m}$ deep (putative layer 5) (Isomura et al., 2009, their Supplementary Fig. 7) and on the antidromic identification of PT neurons projecting to the thalamus or pontine nuclei. Insertions were performed using fine micromanipulators (SM-15 and SMM-200B, Narishige) at least $1 \mathrm{~h}$ before the start of the recording experiment.

The wide-band signals were amplified, filtered (FA64I, Multi Channel Systems; final gain, 2000; bandpass filter, $0.5 \mathrm{~Hz}$ to $10 \mathrm{kHz}$ ) through a 32-channel head-stage (MPA32I, Multi Channel Systems; gain, 10). These signals were digitized at $20 \mathrm{kHz}$ and recorded by three 32 -channel hard-disc recorders (LX-120, TEAC), which simultaneously digitized the pedal positions tracked by angle-encoders and the events of optogenetic stimulation.

In some experiments, the EMG activity of the left forelimb was obtained by an amplifier with a head-stage (EX4-400, Dagan; gain, 1000; bandpass filter, $0.3 \mathrm{~Hz}$ to $10 \mathrm{kHz}$ ). This signal was also digitized at $20 \mathrm{kHz}$ and recorded by the 32-channel hard-disc recorder.

Optical (optogenetic) stimulation. In some sessions, we conducted the Multi-Linc (multiareal/multineuronal light-induced collision) method, which enabled us to effectively identify the pyramidal neurons sending direct projections to specific areas by combining the multiareal optogenetic stimulation and multineuronal recordings. The details of this procedure were described previously (Saiki et al., 2017). Briefly, before the insertion of silicon probes, an optical fiber (FT400EMT, FC, Thorlabs; NA, 0.39; internal/external diameters, $400 / 425 \mu \mathrm{m}$ ) for stimulation was placed on the surface of either M1 or M2, and another optical fiber (FT200EMT, FC, Thorlabs; NA, 0.39; internal/external diameters, 200/ $225 \mu \mathrm{m})$ was vertically inserted into either the thalamus (5000-5500 $\mu \mathrm{m}$ deep) or pontine nuclei (9000 $\mu \mathrm{m}$ deep) using micromanipulators (SM25A, Narishige). To evoke antidromic spikes in specific axonal projections from the IT or PT neurons of M1 or M2 (IT neurons: contralateral M1 or M2; PT neurons: ipsilateral thalamus or pontine nuclei), a blue LED light pulse (intensity, $5-10 \mathrm{~mW}$; duration, $0.5-2 \mathrm{~ms}$, typically $1 \mathrm{~ms}$ ) was applied through each of the two optical fibers using an ultra-highpower LED light source (UHP-Mic-LED-460, FC, Prizmatix) and a stimulator (SEN-8203, Nihon Kohden). To be classified as projecting neurons, the neurons were required to meet several criteria, including constant latency, fixed frequency (frequency-following test, two pulses at $100-250 \mathrm{~Hz}$ ), and collision tests (Lipski, 1981).

The collision test was, at the time, just tentative to readily accumulate spike collision data that would be sufficient for post hoc analysis, completing multineuronal collision tests (Saiki et al., 2017; and see below).

$E M G$ data analysis. The EMG signal was rectified to calculate the laterality index (see below) and onset time of left muscle activity. The onset time was determined by the first of 10 consecutive $1 \mathrm{~ms}$ bins $(10 \mathrm{~ms})$ in which the EMG power deviated by $>5 \mathrm{SD}$ of the mean value calculated during the baseline period $(-1000$ to $-700 \mathrm{~ms}$ relative to the onset of pedal release).

Spike isolation. Raw signal data were processed offline to isolate spike events of individual neurons in each tetrode of the silicon probes. Briefly, spike candidates were detected and clustered by our semiautomatic spike-sorting software, EToS (Takekawa et al., 2010, 2012) (http://etos. sourceforge.net/). The spike clusters were further combined, divided, and/or discarded manually to refine single-neuron clusters, based on the presence or absence of refractory periods $(<2 \mathrm{~ms})$ in their own autocorrelograms and cross-correlograms with other clusters, using the manual clustering software Klusters and the viewing software NeuroScope (Hazan et al., 2006).

Spike collision analysis. To identify IT and PT neurons, we used the Multi-Linc method with post hoc analysis to complete multineuronal collision tests (Saiki et al., 2017). Briefly, after offline sorting for spike isolation, we compared filtered tetrode (four-channel) traces that had no spikes before the stimulus (see Fig. $6 B$, black, control traces) with those that had a spike in one spike cluster (see Fig. $6 \mathrm{~B}$, red test traces) using MATLAB (The MathWorks). If we found antidromic-like (all-or-none and no-jittering; see Fig. $6 \mathrm{~B}$, black arrowheads) spike activities with short latency in many of the control traces, we set a time window for counting possible antidromic spikes, based on a clear dissociation between averaged control and test traces due to presence or absence of spikes. The cutoff threshold defined in a receiver operating characteristic curve for the distribution of most negative points within the time window was used to determine whether the spikes were present or not, so that we obtained spike and no-spike counts in the control and test events. According to this method, we included spike clusters with control spike probability $>50 \%$ and test spike probability less than half of the control. Finally, the passing of the collision test was statistically justified by a $2 \times 2 \chi^{2}$ test $(p<0.05)$ for spike and no-spike counts in control and test events (Saiki et al., 2017, their Supplementary Fig. 9).

The latency of antidromic spikes was defined as the time from the onset of stimulation to the median (the second quartile, 50\%) of their peak positions within the time window, and their jitter was defined as the time between the first $(25 \%)$ and the third $(75 \%)$ quartiles of their peak positions within the time window. In this way, we judged these spikes to be antidromic or not based on collisional disappearance of antidromic spikes (collision test), as well as their all-or-none properties, absence of jitters (constant latency test; $<0.5 \mathrm{~ms}$ ), and high reliability (frequencyfollowing test; if applicable in the tentative collision test).

Analysis of neuronal activity. In each identified/unidentified neuron (spike cluster), basal spiking properties and functional activity in relation to behavioral task performance were analyzed using MATLAB as follows. All spikes occurring for $1 \mathrm{~s}$ after optical stimulation were excluded from spike data for later analyses. The ongoing (all averaged) spike rate and spike duration for individual spike clusters were defined in the same manner as in our previous studies (Isomura et al., 2009; Saiki et al., 2014, 2017).

Electrophysiological studies have indicated differences between the duration of the extracellularly recorded spike waveforms of regularspiking (RS: mostly putative excitatory neurons) and fast-spiking (FS: putative inhibitory neurons) neurons to separate these two classes (Simons, 1978; Bruno and Simons, 2002), and a recent study reported that FS neurons were GABAergic interneurons based on an optical tagging of FS-parvalbumin-positive interneurons (Kim et al., 2016). According to these lines of evidence, spike clusters other than the identified IT and PT neurons were classified as RS and FS neurons based on spike duration ( $\geq 0.6$ ms for RS neurons; see Fig. 2) (Isomura et al., 2009; Saiki et al., 2017). As expected, all IT and PT neurons satisfied the criteria for RS neurons (IT neurons: $1.09 \pm 0.08 \mathrm{~ms}, 0.8-1.2 \mathrm{~ms}$, PT neurons: $1.10 \pm$ $0.09 \mathrm{~ms}, 0.8-1.25 \mathrm{~ms}$ ). Because we had several groups of neurons (RS vs FS, PT vs IT, and M1 vs M2), for simplicity we use abbreviated expressions to refer to each group: for example, we refer to the RS neurons in M1 as M1-RS.

Next, we examined functional (task-related) spike activity in relation to behavioral performance of forelimb movements. We primarily focused on neuronal activity recorded during unilateral (contralateral and ipsilateral) forelimb movements (excluding bilateral movements) to simply interpret the relationship between functional activity and rightleft forelimb control. Thus, spike trains obtained only from unilateral forelimb movement trials were aligned with the onsets $(0 \mathrm{~ms})$ of pedal release (following by $\geq 1$ s holding time) during task performance ( $\geq 20$ trials with total $\geq 250$ spikes). A pedal release event was detected when the pedal was released outside of the holding area (30\% in relative pedal position), and the onset of the pedal release was defined as the nearest time point at which the pedal position exceeded $5 \%$ before the pedal release event. Task-related activity was defined by the task relevance index using the Kolmogorov-Smirnov test, as described previously (Saiki et al., 2014, 2017; Kimura et al., 2017). We defined a task-related neuron as a neuron with a smaller task relevance index than the criterion $(p<$ $10^{-6}$ ) in either a contralateral or ipsilateral trial; task-related neurons were further classified into three categories on the basis of the task relevance index: contralateral (contralateral, $p<10^{-6}$, ipsilateral, $p \geq$ 
A
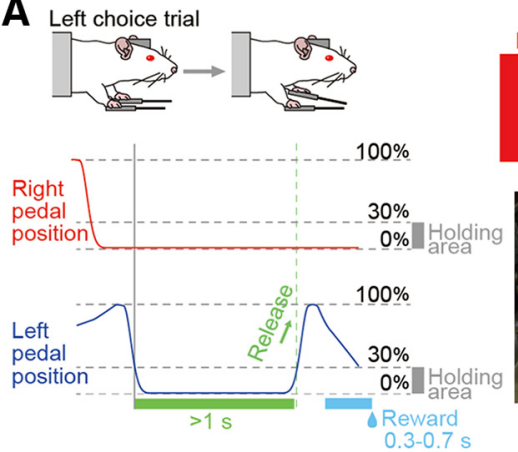

B
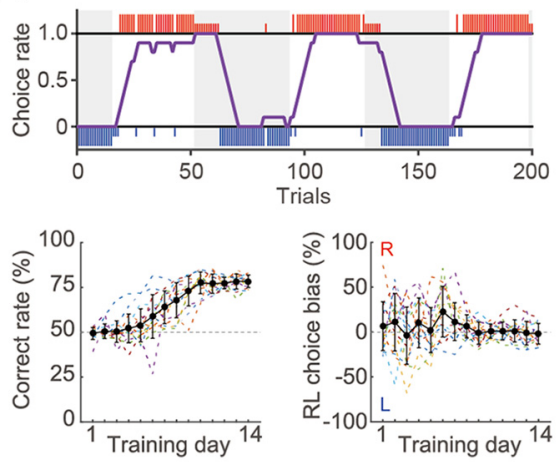

Figure 1. Behavioral task performance. $\boldsymbol{A}$, Schematic diagram of the Right-Left Pedal task. The rats pushed down on both pedals for a short period ( $\geq 1 \mathrm{~s}$ ) to initiate the trial, and then voluntarily chose either of the pedals to receive a reward (e.g., left release). This task consisted of two blocks (the right pedal- and left pedal-rewarded blocks), which were alternated after meeting the criteria (see Materials and Methods). Photographs show the right-left pedal device installed in a stereotaxic frame and a rat manipulating (pushing down) both pedals. $\boldsymbol{B}$, Typical example of task performance and the rats' learning process. Top, Rat chose the correct pedal based on the reward. Large and small colored vertical bars (red represents right choice; blue represents left choice) indicate correct and incorrect trials, respectively. The choice rate of the right pedal (purple line) was calculated by averaging the number of right choices obtained from the past 10 trials. Bottom, 0 training day 14 , the rats chose the rewarded pedal $>75 \%$ (left), and choice bias disappeared throughout the training (right). Solid line indicates average. Dashed lines indicate individual rat. C, Right-left pedal trajectories and EMG activity in left forelimb. Top, Example pedal and EMG traces in three consecutive trials. Bottom, Averaged population EMG power ( \pm SEM) aligned with the onset of pedal release (dashed line).

$10^{-6}$ ), ipsilateral (contralateral, $p \geq 10^{-6}$, ipsilateral, $p<10^{-6}$ ), and bilateral (contralateral, $p<10^{-6}$, ipsilateral, $p<10^{-6}$ ).

We classified task-related neurons as hold-related and go-related using their preferred activities (either contralateral or ipsilateral trials) with the smaller task relevance indices. Premovement activity includes holdrelated and go-related activities, whereas postmovement activities were considered to be go-related. Therefore, we classified the neurons that exhibited their peak-activity timings in the perievent time histograms (PETHs; 20 ms bins) after pedal-release onset as go-related (Post-go-type), and divided the neurons exhibiting their peak-activity timings before pedal-release onset according to the dependence of premovement activity on holding time (see Fig. $3 B, C$ ). We measured each time point crossing the $75 \%$ level of the peak spike activity in all trials for each of the PETHs obtained from different holding time trials (see Fig. 3B, top; $1.0-1.5$, 1.5-2.0, $2.0-2.5$, and 2.5-3.0 s) and estimated the slope in the plot of cross points and holding times (see Fig. $3 B$, bottom). The neurons with slopes $<-0.5$ were classified as hold-related (Hold-type), and otherwise as gorelated (Pre-go-type). Both types of go-related neurons (Pre-go and Post-go-type) were grouped together as Go-type.

To evaluate the laterality of each neuron, we compared the peak activities (maximum firing rate during -1000 to $500 \mathrm{~ms}$ relative to the onset of pedal release) for Go-type, and mean firing rates during holding period $(-1000$ to $0 \mathrm{~ms}$ relative to the onset of pedal release) for Hold-type, between contralateral and ipsilateral trials. In addition, we confirmed the laterality based on normalized peak activities for Go-type neurons, as follows:
Laterality index

$= \begin{cases}(c-i) /(c+i), & \text { if } c>0 \text { and } i>0 \\ +1, & \text { if } c>0 \text { and } i<0 \\ -1, & \text { if } c<0 \text { and } i>0\end{cases}$

where $c$ and $i$ are the activities associated with contralateral and ipsilateral movements, respectively. These parameters were obtained from the following equation:

$$
c, i=F R_{\text {peak }} / F R_{\text {baseline }}-1
$$

where $F R_{\text {peak }}$ is the mean firing rate of the peak period (peak $\pm 150 \mathrm{~ms}$ ), and $F R_{\text {baseline }}$ is the mean firing rate during the baseline period $(-1000$ to $-700 \mathrm{~ms}$ relative to the onset of pedal release). For the laterality index (ranging from -1 to 1 ), values of -1 and 1 indicate ipsilateral and contralateral movement-related neuronal activity, respectively.

The outcome modulation index for the preferred Go-type activity was calculated from the following equation:

Outcome modulation index $=\left(F R_{r}\right.$

$$
\left.-F R_{n r}\right) /\left(F R_{r}+F R_{n r}\right)
$$

where $F R_{r}$ and $F R_{n r}$ is the mean firing rate of the peak period (peak $\pm 150 \mathrm{~ms}$ ) of rewarded (correct) and nonrewarded (error) trials, respectively. If this index is $>0$, the activity is considered positively modulated by outcome.

Histological observations. After the recording experiments, animals were deeply anesthetized with urethane ( $2-3 \mathrm{~g} / \mathrm{kg}$, i.p.; Nacalai Tesque) and perfused transcardially with cold saline followed by $4 \%$ formaldehyde in $0.1 \mathrm{~m}$ phosphate buffer. Whole brains were postfixed and sliced coronally into $50 \mu \mathrm{m}$ serial sections using a microslicer (VT1000S, Leica). The sections were Nissl-stained with Neutral Red (Nacalai Tesque). Optical fiber and electrode tracks were checked in the motor cortices, thalamus, and pontine nuclei under a microscope (BX51N, Olympus).

Experimental design and statistical analyses. In this study, we used 16 adult male W-TChR2V4 rats (Tomita et al., 2009) to investigate the laterality in forelimb-movement representations of M1 and M2 neurons. The W-TChR2V4 rats express ChR2-Venus in neurons ubiquitously throughout the brain (Saiki et al., 2017, their Supplementary Fig. 1A), which is useful for optogenetically evoked spike collisions test to identify IT and PT neurons as descried above. We recorded a total of $834 \mathrm{M1}$ neurons and $1316 \mathrm{M} 2$ neurons during task performance. These neurons were further divided into subclasses: RS and FS neurons (see Fig. 2), task-related and non-task-related neurons (see Fig. 3), IT and PT neurons (see Fig. 7). They were compared using appropriate statistical tests (i.e., one-sample signed-rank test, Mann-Whitney's test, Wilcoxon signed-rank test, $\chi^{2}$ tests, Kolmogorov-Smirnov tests, and two-way ANOVA). These statistical tests were conducted by using MATLAB's Statistics and Machine Learning Toolbox (The MathWorks). Differences were considered statistically significant when $p<0.05$ (see Results). Data in the text and figures are mean \pm SD (unless otherwise noted) and sample number $(n)$.

\section{Results}

\section{Behavioral performance}

To examine the laterality in forelimb-movement representations, we developed a novel behavioral task, the Right-Left Pedal task, in which head-restrained rats correctly manipulated two pedals 
with their forelimbs to obtain a reward (Fig. 1A). Figure $1 B$ (top) shows a typical example of one session of task performance. While switching the blocks, the rats smoothly changed their pedal choices and kept performing the task until they were satisfied with water. In all rats examined, we observed similar performance ( $n=16$ rats, at training day 14: number of performed trials, $1011 \pm 261$ trials $/ 2 \mathrm{~h}$ ).

Population data of learning of the RightLeft Pedal task are shown in Figure $1 B$ (bottom panels). Although rats had a preference for one pedal in the first week (e.g., right), they successfully learned the task paradigm within 2 weeks and properly changed their forelimb movement from one side to the other (i.e., left) within five trials on average with no pedal bias (the number of trials after the block change to achieve the $50 \%$ correct rate, $5 \pm 1$ trials). In our task, the holding area was set $<30 \%$ in relative pedal position. Even if rats moved their forelimb within the holding area, the time was also included as the holding time. Indeed, rats rarely moved forelimbs during the holding period, resulting in small deviation of pedal position (right, $1.0 \pm 1.1 \%$, left, $0.8 \pm$ $1.3 \%)$, which was much smaller compared with criteria for the holding area (30\%). This result indicates that rats pushed both pedals down stably during the holding period (see also EMG activity during the holding time; Fig. $1 C)$.

In some rats ( $n=6$ rats, 15 sessions), we recorded the EMG of the left upper forelimb (Fig. 1C). Figure $1 C$ (top panels) indicates typical traces of the pedal and EMG, in which the left muscles exhibited changes in activity that were associated with movement execution of the left forelimb (left choice trials) but not that of the other forelimb (right choice trials). This was confirmed in all rats examined (change in EMG between pre- and post-movements: right pedal release, $4.4 \pm 8.4 \%$, left pedal release, $40.2 \pm 14.7 \%$ ), and the laterality index of the left muscles activity was -0.56 (Fig. $1 C$, bottom). The onset time of forelimb movement was $-251 \mathrm{~ms}$ relative to the onset of pedal release, indicating that the left muscles became active just before the onset of pedal release. These findings suggest that the rats executed the pedal release using forelimb movement only on the required side.

\section{Functional activity during the forelimb movements in the M1 and $\mathrm{M} 2$ neurons}

Extracellular multineuronal activity was recorded from the output layer (putative layer 5) in M1 and M2 of 16 rats (Fig. 2A) while they performed alternating blocks of contralateral and ipsilateral forelimb choices. We isolated a total of $834 \mathrm{M} 1$ neurons and 1316 M2 neurons from our multineuronal recordings during task performance. These neurons were further classified as RS or FS neurons based on clear bimodality of the spike duration (Fig. 2B), which was consistent with previous reports (Isomura et al., 2009, Saiki et al., 2014, 2017; Kimura et al., 2017).

Next, we analyzed the functional activity in relation to forelimb movements in the M1 and M2 neurons. To define task- related neurons, we calculated the task relevance index from the spike data aligned with the onsets of right or left pedal releases (Fig. 3A) according to previously established methods (Saiki et al., 2014, 2017; Kimura et al., 2017; see Materials and Methods). Figure $3 A$ shows typical examples of one non-task-related (\#1, gray) and three task-related (\#2-4, black) neurons activated before (\#2 and \#3) and after (\#4) forelimb movements. In the premovement functional activities, we observed two types of activity, the first (\#2) showing sustained activity during the holding period, and the other (\#3) phasically activated just before the onset of forelimb movements. To classify these neurons as holdrelated or go-related, we evaluated the dependence of premovement activity on holding time (Fig. $3 B, C$; see Materials and Methods). If a neuron exhibited a dependence on holding time, the estimated slope in the plot of crossing time point versus holding time was negative (Fig. $3 B$, left), whereas the slope was $\sim 0$ when neuronal activity was independent of holding time (Fig. $3 B$, right). Using this method, we observed bimodality of slope histograms in both M1 and M2 (Fig. 3C). Based on a threshold (Fig. $3 C$, vertical lines), we classified neurons as hold-related (Holdtype; $\leq-0.5$ ) or go-related (Pre-go-type; $>-0.5$ ). The Pre-gotype neurons and neurons with their peak-activity timings after the onset of pedal release (Post-go-type) were grouped together as Go-type neurons. In accord with previous studies (Laubach et al., 2000; Narayanan and Laubach, 2009), we also 
A
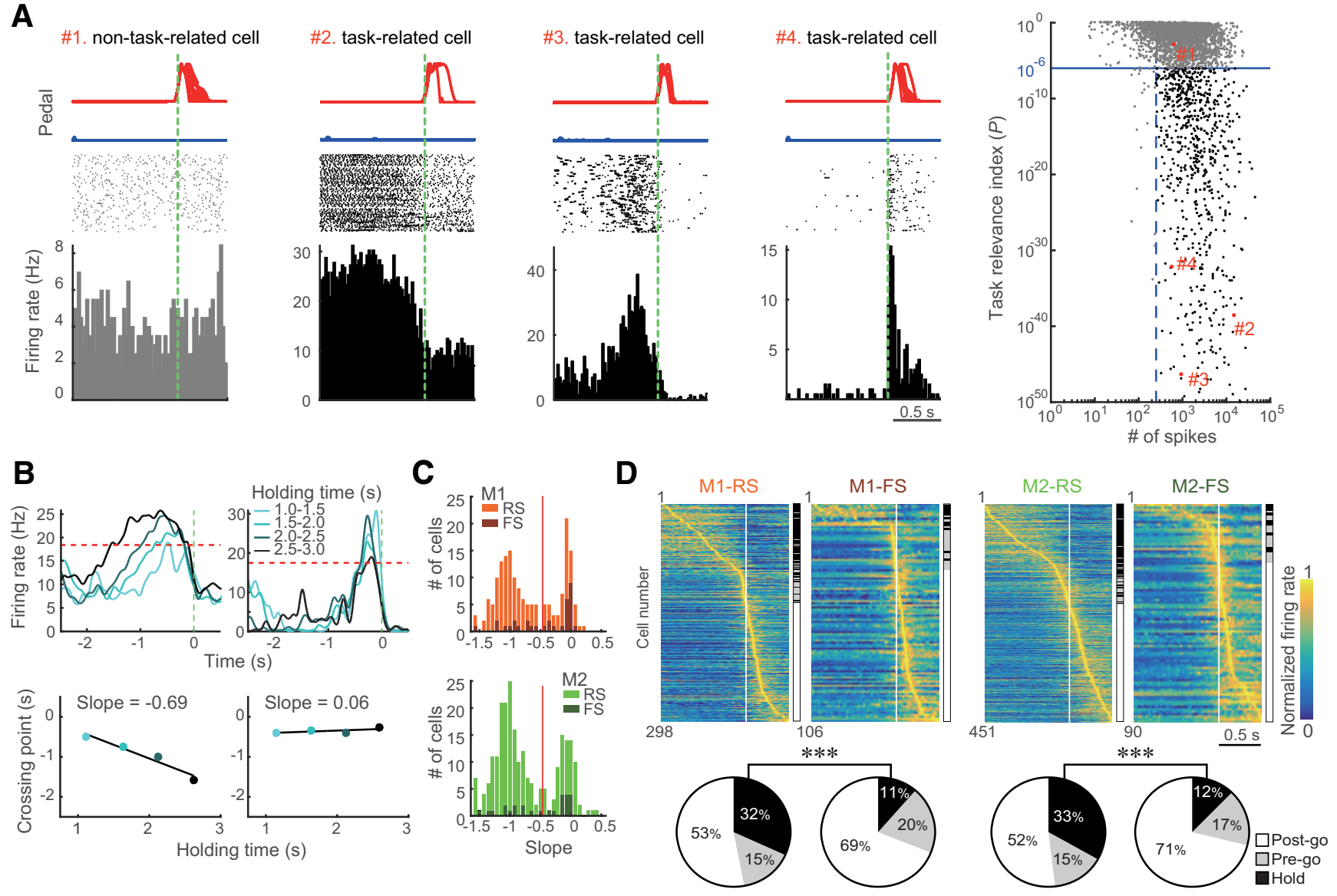

Figure 3. Different types of functional activity in the $\mathrm{M} 1$ and $\mathrm{M} 2$ neurons. $A$, Definition of task-related activity. The number of spikes during contralateral and ipsilateral movement trials was plotted against task relevance index for individual neurons (right scatter plot; see Materials and Methods). Black and gray dots indicate the task-related (blue line, $p<10^{-6}$; blue dashed line, $\geq 250$ spikes) and non-task-related (discarded) neurons, respectively. Red numbers correspond to examples of activities (left panels). Top, Middle, and Bottom, Pedal trajectories, spike raster plots, and PETHs of preferred movement, respectively. Bin width, $20 \mathrm{~ms}$. B, Categorization of Hold-and Pre-go-type activities by dependence on holding time. PETHs calculated from the different holding time trials (top). Intersection with criterion (red dashed line, $75 \%$ of activity in an averaged PETH) was plotted on the holding time, and the slope value of the regression was obtained (bottom). If a neuron had Hold-type-related activity, the slope value was negative (left column). By contrast, the slope value was $\sim 0$ if neuronal activity was independent of holding time (right column). C, Distribution of slope in the motor cortices. Histograms of slopes exhibit a clear bimodality in both M1 and M2.D, Three types of task-related activity in RS and FS neurons in the motor cortices. Top, Each row shows normalized Gaussian-filtered PETH ( $\sigma=12.5 \mathrm{~ms}$, in $0.05 \mathrm{~ms}$ bins) for a single neuron (aligned with the onset of choice: vertical line at $0 \mathrm{~s}$ ). The task-related neurons were sorted by the order of peak time (early to late). Hold-, Pre-go-, and Post-go-type activities are indicated on the right side. Bottom, Population ratios of different activity types for RS and FS neurons in the motor cortices. Black, gray, and white represent Hold-, Pre-go-, and Post-go-type activity, respectively. ${ }^{* * *} p<0.001\left(2 \times 2 \chi^{2}\right.$ tests).

observed the press-related neurons showing higher levels of activity during pedal press than during pedal release. In our task, however, it was difficult to isolate the pedal press onset and to analyze this putative pedal press-related neuron activity due to the variety of movements prior the pedal press. Therefore, we did not focus on the laterality of press-related neurons in this study.

The functional activities of all task-related motor cortices neurons are shown in Figure 3D (top panels) (M1-RS: $n=298$; M1-FS: $n=106$; M2-RS: $n=451$; M2-FS: $n=90$ ). These taskrelated neurons were sorted by peak position (early to late), and the types of functional activity are indicated on the right side. As previously reported (Isomura et al., 2009, 2013; Saiki et al., 2014, 2017; Kimura et al., 2017), we observed different repertoires of task-related activity between RS and FS neurons in the motor cortices. The proportion of Hold-type in RS neurons was larger than that in FS neurons; specifically, the proportion of Go-type in FS neurons was larger (Table 1). The differences were significant in both M1 and M2 (M1: $\chi^{2}=17.0, p<3.8 \times 10^{-5}$; M2: $\chi^{2}=$ 15.9, $p<6.8 \times 10^{-5} ; 2 \times 2 \chi^{2}$ test for Hold: Go ratio between RS and FS; Fig. 3D).
Table 1. Classification of RS and FS neurons ${ }^{a}$

\begin{tabular}{lcclll}
\hline & M1 834 & & & M2 1316 & \\
\cline { 2 - 3 } Total unidentified neurons & RS 677 & FS 157 & & RS 1177 & FS 139 \\
\hline Task-related & $298(100)$ & $106(100)$ & & $451(100)$ & $90(100)$ \\
$\quad$ Hold-type & $95(32)$ & $12(11)$ & & $150(33)$ & $11(12)$ \\
Pre-go-type & $45(15)$ & $21(20)$ & & $67(15)$ & $15(17)$ \\
Post-go-type & $158(53)$ & $73(69)$ & & $234(52)$ & $64(71)$ \\
Non-task-related & 379 & 51 & & 726 & 49 \\
\hline
\end{tabular}

${ }^{a}$ Values are no. (\%).

\section{Laterality in forelimb-movement representations of M1 and $\mathrm{M} 2$}

To investigate the laterality of neuronal activity of motor cortices, we compared PETH for contralateral movements with that for ipsilateral movements. Figure $4 A-F$ indicates the examples of functional activities obtained from contralateral (red) and ipsilateral (blue) movement trials. Here, all examples showed a preferred spike activity during contralateral forelimb movement trials. The activities of M1 Go-type neurons appeared only in association with contralateral pedal choice (Fig. $4 A, B$ ), whereas 
A

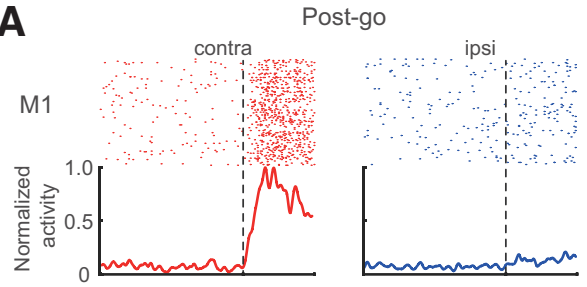

D

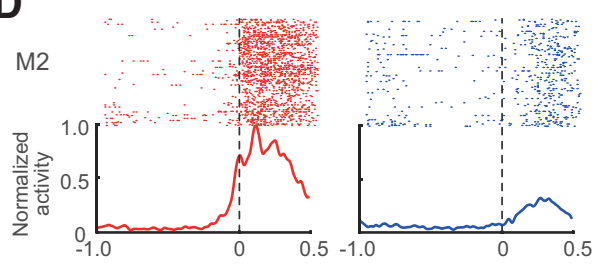

B

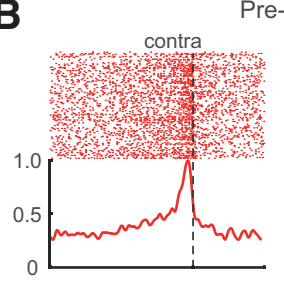

E

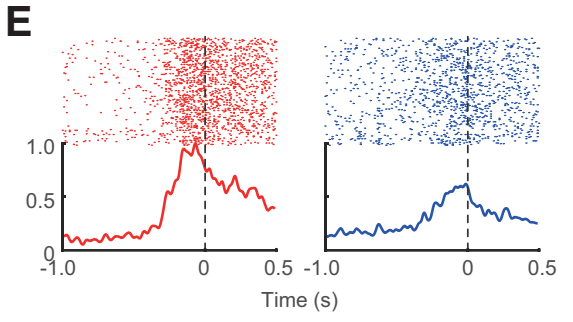

H

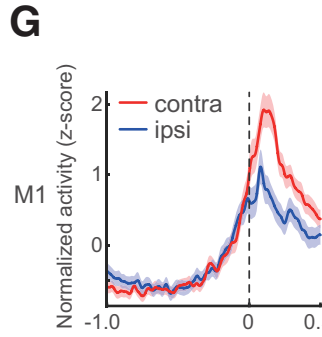

Post-go
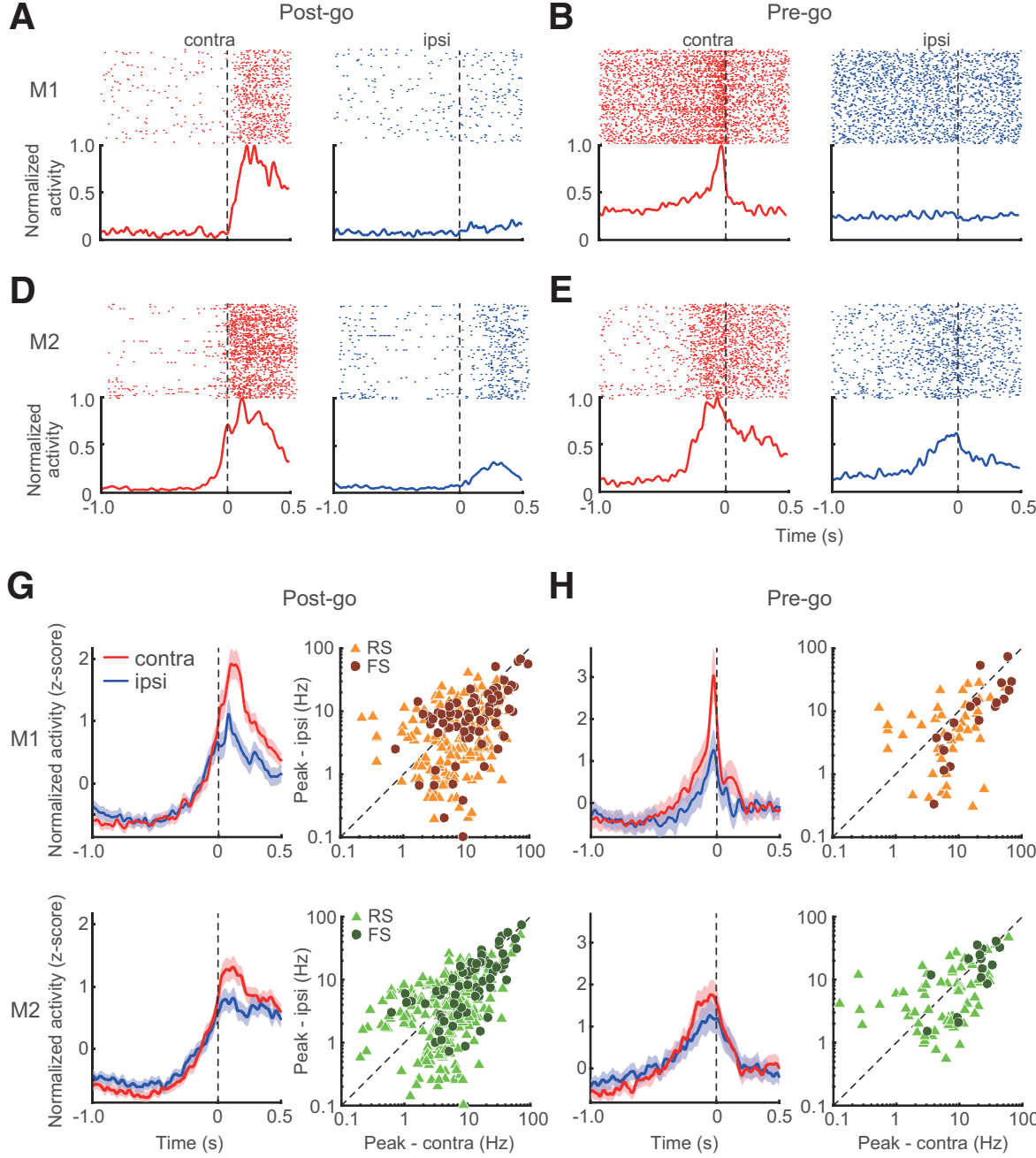

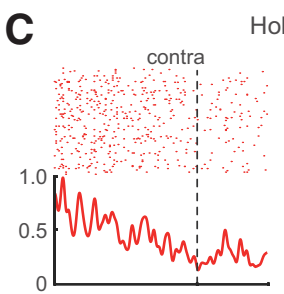

Hold

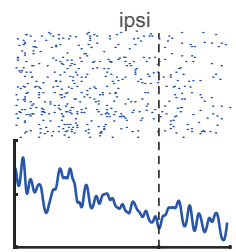

F
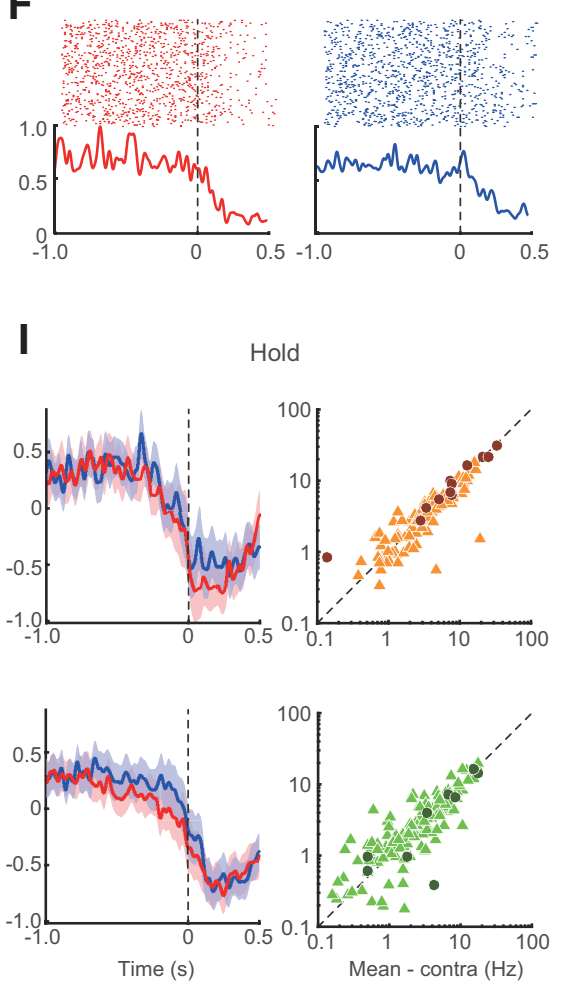

Figure 4. Selectivity of neuronal activity to contralateral and ipsilateral movements in the motor cortices. A-C, Examples of Post-go-, Pre-g0-, and Hold-type functional activities in the M1 neurons in the contralateral (red) or ipsilateral trials (blue). Colored dots and lines indicate spike raster plots and normalized PETHs, respectively. $\boldsymbol{D}-\boldsymbol{F}$, Same as $\boldsymbol{A}-\boldsymbol{C}$ for the M2 neurons. G-I, Population data of three types of functional activity in the M1 (top) and M2 (bottom). Left columns, Averaged PETHs of all Post-go-, Pre-g0-, and Hold-type activities for the contralateral (red) and ipsilateral (blue) choice trials. Shaded regions represent $95 \%$ Cls. Right columns, Peak activity $(\boldsymbol{G}, \boldsymbol{H})$ and mean firing rate (I) of the contralateral (abscissa) and ipsilateral (ordinate) choice trials. Triangles and circles represent individual RS and FS neurons, respectively.

those of M2 appeared during both contralateral and ipsilateral pedal choices (Fig. 4D,E). As for Hold-type activity, similar activities were observed in both contralateral and ipsilateral trials in the $\mathrm{M} 1$ and $\mathrm{M} 2$ (Fig. 4C,F).

To qualitatively show the selectivity of each functional activity in M1 and M2, we normalized and averaged their spike activities (Fig. 4G-I, left columns). Post-go- and Pre-go-type population PETHs revealed that the contralateral preference in the M1 was greater than that of M2 (Fig. 4G,H), whereas Hold-type activity was similar between contralateral and ipsilateral movements in both cortices (Fig. 4I). To quantify these characteristics, we compared the peak activities for Go-type or the mean firing rates before movements for Hold-type (see Materials and Methods) of individual neurons between the contralateral and ipsilateral trials (Fig. 4G-I, right columns). In the motor cortices, Go-type activity was significantly stronger in contralateral trials than ipsilateral ones (M1-RS: contralateral, $10.9 \pm 10.2$ $\mathrm{Hz}$, ipsilateral, $6.8 \pm 7.4 \mathrm{~Hz}, z=5.55, p<2.9 \times 10^{-8}$; M1-FS: contralateral, $23.3 \pm 23.2 \mathrm{~Hz}$, ipsilateral, $14.0 \pm 16.3 \mathrm{~Hz}, z=5.29$, $p<1.2 \times 10^{-7}$; M2-RS: contralateral, $8.2 \pm 9.2 \mathrm{~Hz}$, ipsilateral, $6.1 \pm 7.1 \mathrm{~Hz}, z=4.92, p<8.6 \times 10^{-7}$; M2-FS: contralateral,
$18.1 \pm 15.1 \mathrm{~Hz}$, ipsilateral, $14.7 \pm 14.1 \mathrm{~Hz}, z=2.50, p<1.3 \times$ $10^{-2}$, Wilcoxon signed-rank test), but there were a considerable number of ipsilateral biased and nonbiased neurons in M2 rather than in M1 (i.e., M2 had a much weaker contralateral bias of forelimb movements than M1). This observation was confirmed by a direct comparison of contralateral/ipsilateral difference of activities between M1 and M2 in both RS and FS neurons (M1RS: $4.1 \pm 10.4 \mathrm{~Hz}$; M2-RS: $2.1 \pm 7.0 \mathrm{~Hz} ; z=2.54, p<1.1 \times 10^{-2}$; M1-FS: $9.3 \pm 16.3 \mathrm{~Hz}$; M2-FS: $3.4 \pm 9.7 \mathrm{~Hz} ; z=2.52, p<1.2 \times$ $10^{-2}$, Mann-Whitney's test). As for Hold-type activity, both areas exhibited no bias of laterality (M1-RS: contralateral, $6.3 \pm 5.4$ $\mathrm{Hz}$, ipsilateral, $5.4 \pm 4.7 \mathrm{~Hz}, z=1.35, p=0.18$; M1-FS: contralateral, $15.7 \pm 11.8 \mathrm{~Hz}$, ipsilateral, $14.9 \pm 11.8 \mathrm{~Hz}, z=0.08, p=$ 0.97; M2-RS: contralateral, $5.8 \pm 5.0 \mathrm{~Hz}$, ipsilateral, $5.4 \pm 4.6 \mathrm{~Hz}$, $z=1.07, p=0.28$; M2-FS: contralateral, $12.0 \pm 7.9 \mathrm{~Hz}$, ipsilateral, $11.1 \pm 9.4 \mathrm{~Hz}, z=0.18, p=0.90$, Wilcoxon signed-rank test) and no significant difference between M1 and M2 (M1-RS: $-0.1 \pm 2.0 \mathrm{~Hz}$; M2-RS: $0.8 \pm 1.9 \mathrm{~Hz} ; z=-0.95, p=0.34$; M1-FS: $0.4 \pm 2.2 \mathrm{~Hz}$; M2-FS: $-0.1 \pm 1.6 \mathrm{~Hz} ; z=-0.95, p=0.34$, Mann-Whitney's test). 
A

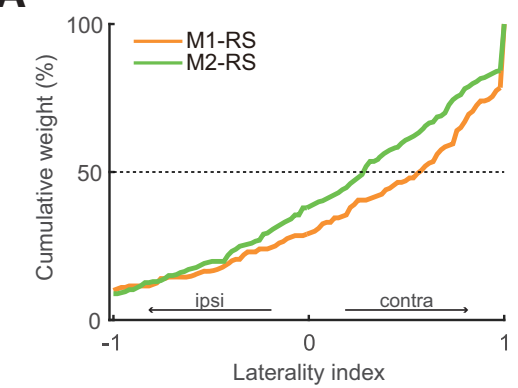

C

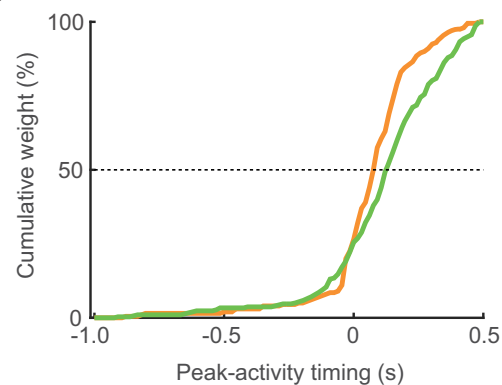

E

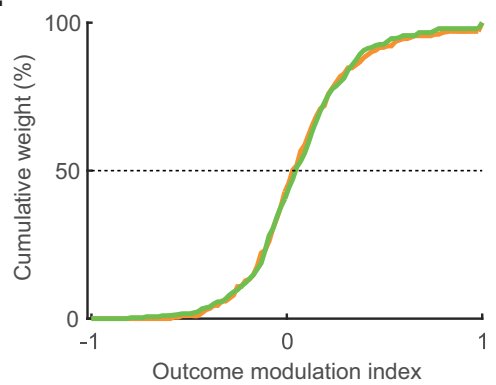

B

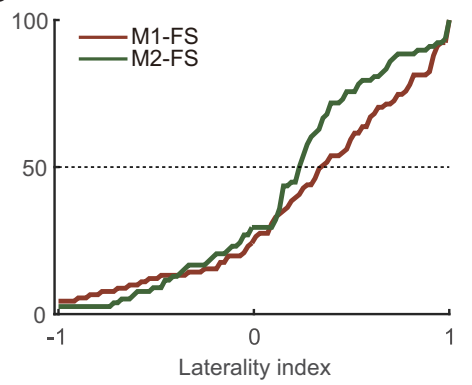

D

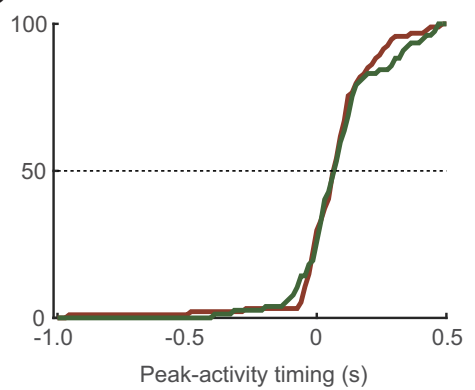

$\mathbf{F}$

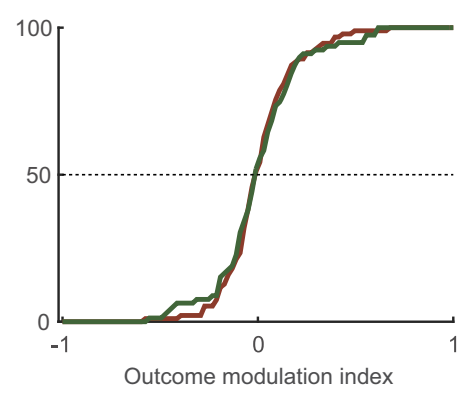

Figure 5. Laterality indices, peak-activity timing, and outcome modulation indices of Go-type activity in M1 and M2 neurons. $\boldsymbol{A}, \boldsymbol{B}$, Cumulative distributions of the laterality indices for $\mathrm{RS}(\boldsymbol{A})$ and $\mathrm{FS}(\boldsymbol{B})$ neurons in the M1 and M2. Positive and negative values indicate greater activity for contralateral and ipsilateral movements, respectively. M1 neurons preferentially represented the contralateral forelimb movements to a greater extent than M2 neurons (rightward shift). C, $\boldsymbol{D}$, Cumulative distributions of the peak-activity timing for $\mathrm{RS}(\boldsymbol{C})$ and $\mathrm{FS}(\boldsymbol{D})$ neurons. $\boldsymbol{E}, \boldsymbol{F}$, Cumulative distributions of the outcome modulation indices for $\mathrm{RS}(\boldsymbol{E})$ and FS $(\boldsymbol{F})$ neurons. Positive and negative values indicate greater activity for rewarded and nonrewarded trials, respectively (see Materials and Methods).

In addition to the comparison of the raw firing rates, we evaluated the laterality index based on the normalized firing rate for phasic activity observed in Go-type neurons (see Materials and Methods). Figure $5 A$ indicates the distribution of laterality indices in the RS Go-type neurons. The laterality indices were biased toward contralateral preference in both areas (Table 2), but M1 neurons preferentially represented the contralateral forelimb movements to a greater extent than M2 neurons (rightward shift), and this difference was statistically confirmed by directly comparing the laterality indices between M1 and M2 (M1-Go: $n=203$, M2-Go: $n=301, z=2.43, p<1.52 \times 10^{-2}$, MannWhitney's test). Similar to RS neurons, M1-FS neurons had a greater contralateral bias in comparison with M2-FS neurons (Fig. 5B; Table 2), but we did not observe a significant difference (M1-Go: $n=94$, M2-Go: $n=79, z=1.54, p=0.12$, MannWhitney's test).

We examined the whole dependence by two-way ANOVA with factors of area (M1 vs M2) and cell-type (RS vs FS), including other viewpoints: peak-activity timing (Evarts, 1973; Narayanan and Laubach, 2009) and outcome modulation (Georgopoulos et al., 1982; Laubach et al., 2000) as well as the laterality. We confirmed significant main effects of area and cell-type in the laterality index (Fig. 5A,B; Table 2). Outcome modulation index exhibited a significant main effect of cell-type but not area (Fig. $5 E, F$; Table 2), indicating the greater positive outcome modulation of RS neurons than FS neurons in both areas. By contrast, we did not find any main effects in the peak-activity timing (Fig. $5 C, D$; Table 2). Also, we found no clear correlations among the laterality index, peak-activity timing, and outcome modulation index (data not shown).

\section{Motor laterality in the IT and PT neurons}

Finally, we investigated how two classes of cortical projection neurons in the output layer, IT and PT neurons, participate in the behavioral functions. Identification of these neuron classes was conducted using the Multi-Linc method (Saiki et al., 2017) with antidromic stimulation of the contralateral motor cortex (cM1 or cM2) for IT neurons and of the ipsilateral ventral nuclei of thalamus or pontine nuclei for PT neurons (Fig. 6A). Figure $6 B$ shows the typical tetrode traces of antidromic spikes (black) and their disappearance due to collision with spontaneous spikes (red) in IT (top; M2 to cM2) and PT (bottom; M1 to iPn) neurons. The reconstructed position of identified IT and PT neurons revealed both were located at a similar cortical depth $(z=0.77, p=0.44$, MannWhitney's test; Fig. 6C). This indicates intermingled existence of IT and PT neurons as reported in previous studies (Catsman-Berrevoets et al., 1980; Reiner et al., 2003; Morishima and Kawaguchi, 2006). We confirmed that the latency of antidromic spikes was shorter in PT neurons (e.g., Fig. $6 B$, bottom) than in IT neurons (e.g., Fig. 6B, top) in both M1 and M2 at a population level (Fig. $6 D$; M1-IT: $n=30,11.5 \pm 3.6 \mathrm{~ms}$, M1PT: $n=54,6.0 \pm 2.7 \mathrm{~ms}, z=5.80, p<6.7 \times 10^{-9}$; M2-IT: $n=$ 74, $11.6 \pm 3.5 \mathrm{~ms}$, M2-PT: $n=89,7.4 \pm 3.1 \mathrm{~ms} ; z=6.53, p<$ $6.2 \times 10^{-11}$, Mann-Whitney's test), consistent with previous studies (Mallet et al., 2006; Li et al., 2015; Saiki et al., 2017). In addition, we found that the antidromic spike latency of PT neurons was significantly shorter in M1 than in M2 $(z=2.85, p<$ $4.3 \times 10^{-3}$, Mann-Whitney's test), but not in IT neurons $(z=$ $0.43, p=0.67)$.

The functional activity of identified IT and PT neurons was further analyzed according to the method described above (Fig. 7). Of the 247 identified projection neurons, 140 were classified as task-related neurons (Fig. 7A; Table 3). Consistent with the results from unidentified RS neurons (Fig. 3D), both the IT and PT neuron groups contained three types of functional activity (Table 3). Although the fractions of PT neurons were different from those of IT neurons, we could not find significant difference between IT and PT neurons (M1: $\chi^{2}=0.34, p=0.56$; M2: $\chi^{2}=$ $0.16, p=0.69,2 \times 2 \chi^{2}$ test for Hold: Go ratio; Fig. $7 A$ ). 
Table 2. Comparison of laterality index, peak-activity timing, and outcome modulation index in RS and FS neurons by two-way ANOVA

\begin{tabular}{|c|c|c|c|c|c|c|}
\hline & \multicolumn{4}{|l|}{ Mean (SEM) } & & \\
\hline & \multicolumn{2}{|l|}{ M1 } & \multicolumn{2}{|l|}{ M2 } & \multicolumn{2}{|l|}{$p$ value ( $F$ value) } \\
\hline & RS & FS & RS & FS & Area (M1 vs M2) & Cell type (RS vs FS) \\
\hline Laterality index & $0.32(0.05)$ & $0.31(0.06)$ & $0.19(0.04)$ & $0.21(0.05)$ & $1.23 \times 10^{-3}(10.53)$ & $4.72 \times 10^{-2}(3.95)$ \\
\hline Peak-activity timing (ms) & $86.5(13.6)$ & $80.4(17.7)$ & $131.6(13.3)$ & $107.4(19.7)$ & $0.10(2.78)$ & $0.92(0.01)$ \\
\hline Outcome modulation index & $0.09(0.02)$ & $0.03(0.02)$ & $0.09(0.02)$ & $0.02(0.03)$ & $0.69(0.16)$ & $8.50 \times 10^{-3}(6.97)$ \\
\hline
\end{tabular}

Next, task-related IT and PT neurons were classified into three categories on the basis of task relevance index (contralateral, red; ipsilateral, blue; bilateral, gray; see Materials and Methods). The proportion of contralateral neurons in PT neurons was larger than that in IT neurons [Figure 7B; M1-PT: contralateral, $n=17$ (65\%), ipsilateral, $n=2(8 \%)$, bilateral, $n=7$ (27\%); M1-IT: contralateral, $n=5$ (31\%), ipsilateral, $n=4$ (25\%), bilateral, $n=7$ (44\%); M2-PT: contralateral, $n=$ $18(42 \%)$, ipsilateral, $n=10$ (23\%), bilateral, $n=15$ (35\%); M2-IT: contralateral, $n=11$ (20\%), ipsilateral, $n=12(22 \%)$, bilateral, $n=32(58 \%)$ ], whereas the unidentified RS neurons of M1 and M2 (Fig. $7 B$, inset) yielded intermediate results, as expected [M1-RS: contralateral, $n=127$ (43\%), ipsilateral, $n=67$ (22\%), bilateral, $n=104$ (35\%); M2-RS: contralateral, $n=$ 178 (39\%), ipsilateral, $n=126$ (28\%), bilateral, $n=147(33 \%)]$. The differences in contralateral proportion between IT and PT neurons were significant in both $\mathrm{M} 1$ and M2 (M1: $\chi^{2}=4.63, p<3.2 \times 10^{-2}$; M2: $\chi^{2}=5.5, p<1.9 \times 10^{-2}, 2 \times 2 \chi^{2}$ test for contralateral vs other neurons).

To quantify the laterality of the IT and PT neurons, we assessed the peak activity (for Go-type; Fig. 7C, right column) and mean firing rate (for Hold-type; Fig. $7 C$, left column). As observed in the unidentified neurons (Fig. 4I), Hold-type neurons exhibited neither contralateral nor ipsilateral bias in M1 and M2 (M1-IT: contralateral, $5.9 \pm 6.0 \mathrm{~Hz}$, ipsilateral, $4.8 \pm 4.2 \mathrm{~Hz}, z=0.94, p=0.44$; M1-PT: contralateral, $3.9 \pm 2.3 \mathrm{~Hz}$, ipsilateral, $4.2 \pm 2.2 \mathrm{~Hz}, z=0.52, p=0.69$, M2-IT: contralateral, $3.8 \pm 3.8$ $\mathrm{Hz}$, ipsilateral, $3.8 \pm 4.0 \mathrm{~Hz}, z=0.11, p=0.93$; M1-PT: contralateral, $4.1 \pm 1.9 \mathrm{~Hz}$, ipsilateral, $4.1 \pm 1.8 \mathrm{~Hz}$, not significant, Wilcoxon signed-rank test). Consistent with the analysis of proportion (Fig. 7B), M1-PT exhibited significantly greater activity in contralateral than ipsilateral movements (contralateral, $10.6 \pm 6.4 \mathrm{~Hz}$, ipsilateral, $7.0 \pm 6.1 \mathrm{~Hz}, z=2.17, p<3.0 \times 10^{-2}$, Wilcoxon signed-rank test), whereas M1-IT, M2-PT and M2-IT did not (M1-IT: contralateral, $12.1 \pm 12.1 \mathrm{~Hz}$, ipsilateral, $4.6 \pm$ $3.3 \mathrm{~Hz}, z=1.95, p=0.05$; M2-PT: contralateral, $8.5 \pm 7.1 \mathrm{~Hz}$, ipsilateral, $7.5 \pm 7.3 \mathrm{~Hz}, z=1.35, p=0.18$; M2-IT: contralateral, $7.9 \pm 10.1 \mathrm{~Hz}$, ipsilateral, $7.0 \pm 5.0 \mathrm{~Hz}, z=0.38, p=0.71$, Wilcoxon signed-rank test). Furthermore, we confirmed the laterality of Go-type neurons with the normalized laterality indices.
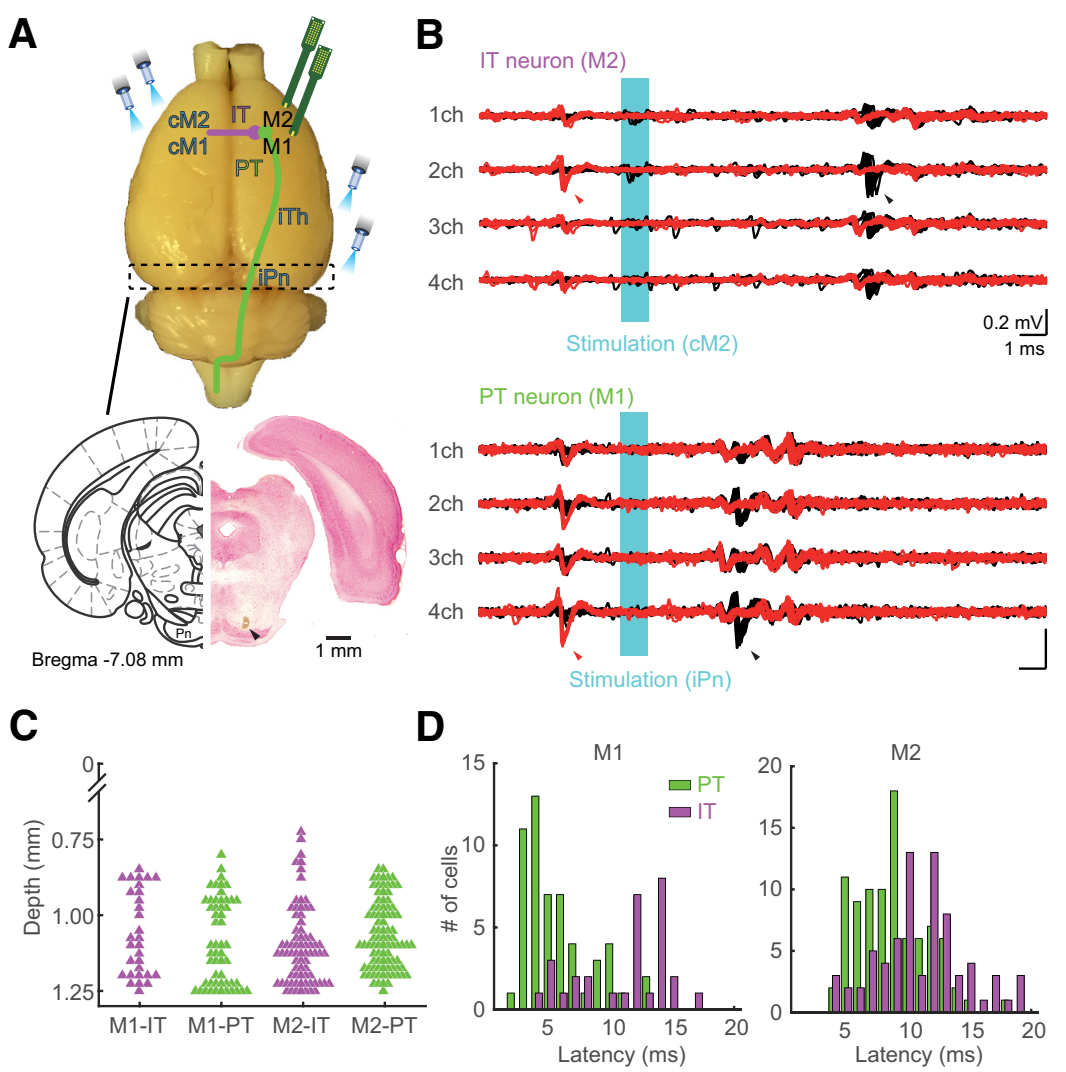

Figure 6. Identification of IT and PT neurons. $\boldsymbol{A}$, Top, Schema showing the position of optical fibers for identifying the IT and PT neurons. The contralateral motor cortex (CM1 or cM2), and either ipsilateral ventral nuclei of thalamus (iTh) or pontine nuclei (iPn) were stimulated for identification of IT and PT neurons, respectively. Bottom, Stimulation site for identification of PT neuron. Track of an optical fiber (arrowhead) into the iPn in a Nissl-stained section. $\boldsymbol{B}$, Examples of recordings from IT (top) and PT (bottom) neurons during optical stimulation (cyan area), with spike collisions. Black and red traces represent the antidromic spikes to optical precedence of spontaneous spikes used as triggers for optical stimulation in collision tests. $\boldsymbol{C}$, Reconstructed recording position of neurons enabled to specify putative layer 5 (see Materials and Methods). D, Distribution of spike latency after antidromic stimulation in IT (purple) and PT (green) neurons of M1 (left) and M2 (right).

The laterality indices of PT Go-type neurons in both areas were significantly greater than zero (M1-PT: $z=2.24, p<2.5 \times 10^{-2}$; M2-PT: $z=2.28, p<2.2 \times 10^{-2}$, one-sample signed-rank test; Table 4) but not in IT Go-type neurons (M1-IT: $z=0.16, p=0.91$; M2-IT: $z=0.21, p=0.83$; Table 4 ). The distributions of laterality indices of PT neurons tended to be more biased toward contralateral movements (rightward shift) than those of IT neurons in both motor cortices (Fig. 7D; M1: $z=0.33, p=0.74 ; \mathrm{M} 2: z=1.44, p=0.15$, Mann-Whitney's test), and this difference in laterality indices between IT and PT was statistically confirmed by two-way ANOVA (Table 4).

At last, we summarized the laterality indices of identified IT and PT neurons in M1 and M2 (Fig. 8). Consistent with primate studies, M1 neurons are more biased to contralateral movements 
A
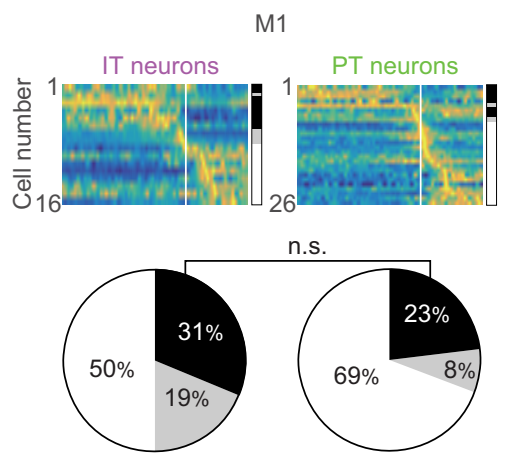

B
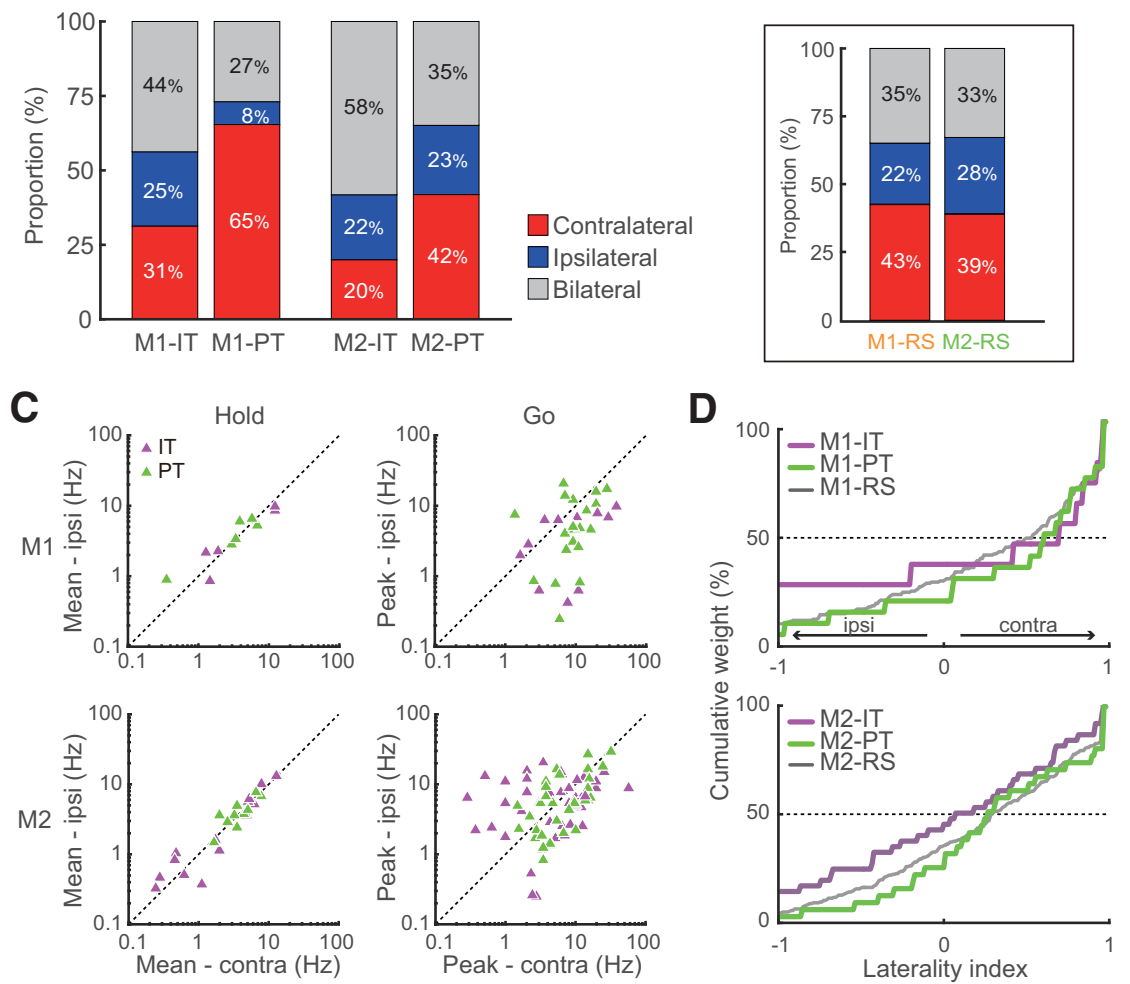

Figure 7. Functional activity and motor laterality of IT and PT neurons. A, Functional activity (top) and fraction (bottom) of IT and PT neurons in M1 and M2. Legend is the same as Figure 3D. B. Proportion of neurons selective for contralateral, ipsilateral, or bilateral movement. Stacked bar graphs represent the ratios of selectivity in IT and PT neurons in the motor cortices. Each category is color-coded: red represents contralateral neurons; blue represents ipsilateral neurons; gray represents bilateral neurons. Insets, Same as $\boldsymbol{B}$ for unidentified RS neurons in the M1 and M2. C, Mean activity (for Hold-type; left column) and peak activity (for Go-type; right column) of the contralateral and ipsilateral movement trials in the IT (purple) and PT (green) neurons. D, Cumulative distributions of the laterality indices for IT and PT neurons in M1 (top) and M2 (bottom). Gray lines are the same as Figure $5 \mathrm{~A}$.

Table 3. Classification of identified IT and PT neurons ${ }^{a}$

\begin{tabular}{lcclll}
\hline & \multicolumn{2}{l}{ M1 84 } & & \multicolumn{2}{l}{ M2 163 } \\
\cline { 2 - 3 } \cline { 6 - 6 } Total identified neurons & IT 30 & PT 54 & & IT 74 & PT 89 \\
\hline Task-related & $16(100)$ & $26(100)$ & & $55(100)$ & $43(100)$ \\
Hold-type & $5(31)$ & $6(23)$ & & $15(27)$ & $11(25)$ \\
Pre-g0-type & $3(19)$ & $2(8)$ & & $7(13)$ & $11(26)$ \\
Post-go-type & $8(50)$ & $18(69)$ & & $33(60)$ & $21(49)$ \\
Non-task-related & 14 & 28 & & 19 & 46 \\
\hline
\end{tabular}

Values are no. (\%).

than M2 neurons. In addition, PT neurons were more biased to contralateral than IT neurons in both areas. Consequently, M1-PT neurons showed a greatest contralateral bias, and M2-IT neurons exhibited little bias. It suggests the M1-PT neurons pre- dominantly drive contralateral movements, whereas the M2-IT neurons coordinate contralateral and ipsilateral movements in a counterbalanced manner. These different neurons may cooperatively participate in bimanual coordination of forelimb movements.

\section{Discussion}

To investigate the motor laterality in the two distinct motor cortices (M1 and M2) and projection cells (IT and PT neurons), neuronal activity was recorded while the rats performed the Right-Left Pedal task. The main results are summarized as follows: (1) the proportion of Go-type and Hold-type activity was different between the RS and FS neurons; (2) Go-type neurons in M1 preferentially represented contralateral forelimb movements to a greater extent than those in M2, whereas Hold-type neurons exhibited no lateral bias; and (3) the PT neurons represented contralateral movements more preferentially than IT neurons in both M1 and M2 (Fig. 8).

\section{Novel behavioral task to search for motor laterality in rodents}

Several sophisticated studies in primates revealed the hierarchy of motor laterality; in these works, great care was taken to train animals to use only the required part of the limb, resulting in specific activation of targeted muscles (e.g., Tanji et al., 1987, 1988). Currently, rodents are more useful to investigate the motor laterality because they make it possible to examine not only neuronal activity for specific behaviors, but also the network mechanism and even causality, based on genetic identification and manipulation of the neuronal/network activity (Li et al., 2016; Tantirigama et al., 2016; Saiki et al., 2017). Notwithstanding the huge advantages of these techniques, to date, motor laterality has remained poorly understood in rodents. This is because most behavioral experiments in rodents are conducted using their locomotion under freely moving conditions (Kitsukawa et al., 2011; Soma et al., 2014; Kawai et al., 2015), in which it is difficult to precisely measure discrete movements of individual forelimbs in a steady posture and at high spatiotemporal resolution.

On the other hand, a head-fixed condition would aid in such precise measurement of forelimb movements by avoiding locomotion and effectively maintaining a steady posture. Indeed, several studies using head-fixed rodents revealed neuronal representations of motor information for a single forelimb with high spatiotemporal resolution [e.g., motor preparation and execution (Isomura et al., 2009; Saiki et al., 2014), motor acquisition (Masamizu et al., 2014; Peters et al., 2014), and kinematic information (Panigrahi et al., 2015; Yttri and Dudman, 2016)]. In addition, a recent study using mice revealed the direction, but not 
Table 4. Comparison of laterality index, peak-activity timing, and outcome modulation index in IT and PT neurons by two-way ANOVA

\begin{tabular}{|c|c|c|c|c|c|c|}
\hline & \multicolumn{4}{|l|}{ Mean (SEM) } & & \\
\hline & \multicolumn{2}{|l|}{ M1 } & \multicolumn{2}{|l|}{ M2 } & \multicolumn{2}{|l|}{$p$ value (Fvalue) } \\
\hline & IT & PT & IT & PT & Area (M1 vs M2) & Cell type (IT vs PT) \\
\hline Laterality index & $0.24(0.26)$ & $0.41(0.15)$ & $0.01(0.11)$ & $0.26(0.11)$ & $0.08(3.19)$ & $2.10 \times 10^{-2}(5.50)$ \\
\hline Peak-activity timing (ms) & $55.3(98.5)$ & $129.6(34.5)$ & $115.6(42.6)$ & $86.7(38.4)$ & $9.14 \times 10^{-3}(7.07)$ & $0.08(3.08)$ \\
\hline Outcome modulation index & $-0.02(0.05)$ & $0.13(0.05)$ & $0.07(0.04)$ & $0.11(0.03)$ & $0.26(1.30)$ & $0.51(0.43)$ \\
\hline
\end{tabular}

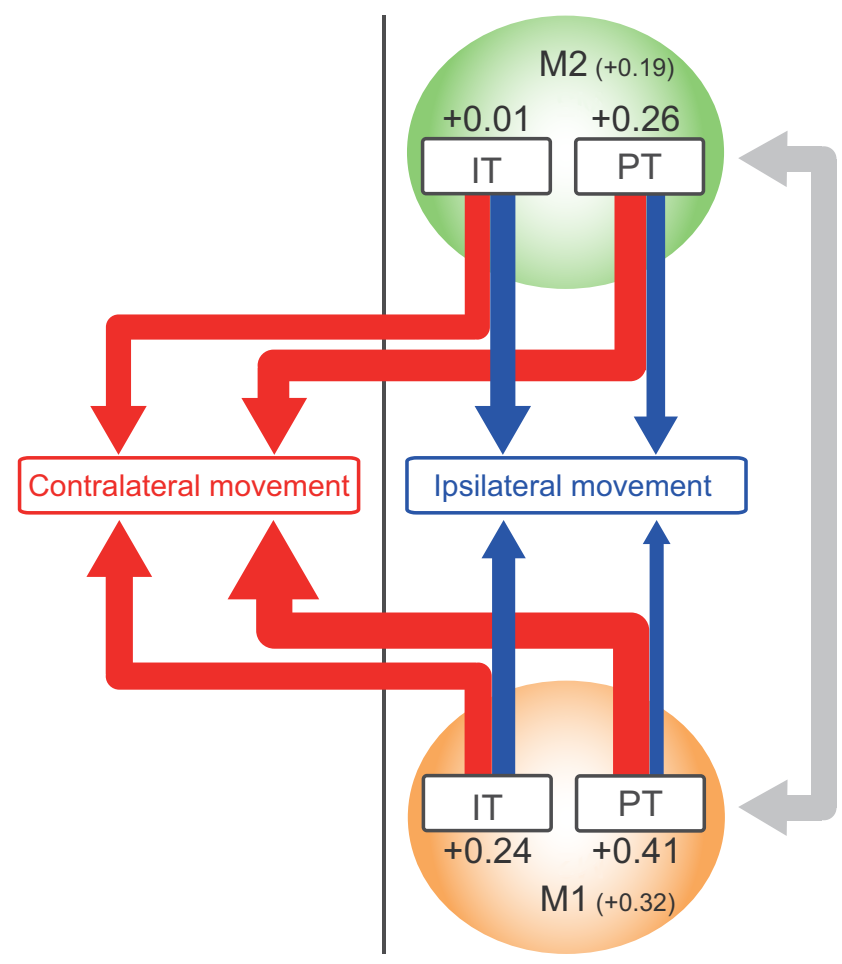

Figure 8. Schematic summary of laterality in motor representation in IT and PT neurons in M1 and M2. Red and blue arrows indicate neuronal activity for contralateral and ipsilateral movements, respectively. The relative strength is indicated by thickness of arrow. The number indicates averaged laterality index for each neuron type. The M1 and M2 neurons may cooperatively communicate with each other to control the movements (gray arrow).

laterality, representation of tongue movements in PT and IT neurons of the anterior lateral motor cortex (Li et al., 2015). However, the motor laterality of forelimb movements has not been examined thus far, largely because there was no adequate behavioral system for monitoring movements of right and left forelimbs separately under head-fixation condition. In this study, we developed the Right-Left Pedal task, in which head-restrained rats manipulated two pedals individually with the right or left forelimb, under a steady posture and without locomotion. Using this system, we observed distinct motor laterality between M1 and M2, as well as between IT and PT neurons. Thus, in combination of cutting-edge techniques, our system could be used to elucidate the neuronal basis of motor laterality and coordination. In addition, our system might be applicable to study other issues of the motor cortex, such as motor acquisition (Kargo and Nitz, 2003, 2004) and kinematic information (Lambercy et al., 2015) in a steady posture and at high spatiotemporal resolution.

\section{Functional repertoire of motor cortex neurons}

Previous studies assessed the spike properties of the M1 and M2 neurons (Isomura et al., 2009; Kimura et al., 2017), revealing similar functional repertoires of RS neurons in rats manipulating a lever with the contralateral forelimb. For example, Saiki et al. (2014) reported that there are no major differences between M1 and M2 neurons in the basal spiking properties and functional activities of voluntary forelimb movements. Consistent with that, we obtained similar observations in M1 and M2 neurons, including similar basal spiking properties (data not shown) and fractions of activity types (Fig. 3D). Thus, we confirmed that we recorded neuronal activity certainly in the forelimb area of M2 as well as M1. Despite such similar functional characteristics of M1 and M2 neurons for one forelimb, we found large differences between the two areas in motor laterality for movements of right and left forelimbs (for further discussion, see below).

In this study, we classified premovement activity as go-related or hold-related based on the dependence of the activity on holding time. This classification revealed a clear bimodal distribution (Fig. 3C). The go-related activity (independent of holding time) preceded the onset of pedal release and was then rapidly decreased during movement expression in a phasic manner, and it is most likely to be involved in motor preparation or initiation. Holdtype activity can be involved in motor preparation/planning or stillness. Although we observed similar fractions of Hold-type activity in M1 and M2, it should be noted that the Hold-type activity of M2-RS, but not M1-RS, quickly decreased when the holding time was extended by a cue presentation (i.e., this was not simple stillness-related activity) (Saiki et al., 2014). This suggests that rodent M2 might be more related to preparation/planning than M1 (Narayanan and Laubach, 2009; Li et al., 2015, 2016), in accordance with primate studies (for review, see Shenoy et al., 2013).

\section{Distinct laterality in $\mathrm{M} 1$ and $\mathrm{M} 2$ representations}

We quantitatively revealed that two distinct motor areas in rodent represented different laterality information in a cell-type specific manner. Consistent with primate studies (Kurata, 2010; Nakayama et al., 2015), the M1 neurons showed greater contralateral bias than M2 neurons (Figs. 4, 5). Although there were several studies that looked similar to ours at first glance, in that RS and FS neurons were activated by contralateral and bilateral movements, respectively, these studies revealed only the direction, but not laterality, representation for effector organ movements (primate: prefrontal cortex neurons representing eye saccades, Johnston et al., 2009; rodent: anterior lateral motor cortex neurons representing tongue movements, Li et al., 2015). Therefore, this is the first report of laterality in forelimb-movement representations of motor cortex neurons in rodents.

Many studies support the idea that rodent M2 is homologous to the primate PM/SMA, based on the projection patterns and the motor response to intracortical microstimulation (Donoghue and Wise, 1982; Neafsey et al., 1986; Reep et al., 1987; Deffeyes et al., 2015). In primates, laterality preference is one feature used to divide motor cortices into M1 and higher-order motor related areas (Tanji et al., 1987; Cisek et al., 2003; Kurata, 2010; Nakayama et al., 2015). Other cortical areas also exhibit laterality preference in 
primates (Kermadi et al., 2000; Chang et al., 2008; Chang and Snyder, 2012; Nakayama et al., 2015). For example, the cingulate motor area has bilateral neurons much more than the posterior parietal cortex (Kermadi et al., 2000), and the cingulate motor area neurons showed less biased laterality than the SMA neurons (Nakayama et al., 2015). However, it remains unknown whether this similar relationship exists in rodents. Here, we demonstrated that motor laterality exhibited a clear difference between these areas (i.e., that M1 neurons have greater contralateral preference than M2 neurons) (Figs. 4, 5). In addition to histological and functional comparisons (Neafsey et al., 1986; Rouiller et al., 1993; Saiki et al., 2014; Hira et al., 2015), our findings support the idea that rodent motor cortices have a functional diversity in which M2 represents higher-order information about movements, whereas M1 encodes concrete motor information, such as motor commands to the musculoskeletal system.

\section{Distinct laterality in representations of IT and PT neurons}

Consistent with previous studies (Mallet et al., 2006; Li et al., 2015; Saiki et al., 2017), we observed that the latency of antidromic spikes was significantly shorter in PT neurons than in IT neurons (Fig. 6D), indicating the reliability of our antidromic identification using the spike collision test. Similar to primate M1-PT and PM-PT neurons (Kraskov et al., 2009), we found that the antidromic spike latency of PT neurons was significantly shorter in M1 than in M2, again supporting the idea that rodent M2 is homologous to primate PM.

We examined the motor laterality of IT and PT neurons, as well as unidentified RS neurons, and found that Go-type PT neurons exhibited bias toward contralateral preference, whereas IT neurons exhibited no bias in both M1 and M2 (Fig. 7). We also observed the larger fraction of contralateral preference in M1-PT (65\%) than M2-PT (42\%). A previous study in primate, in which PT neurons were identified with antidromic stimulation to the medullary pyramid, reported that PT neurons in the higherorder motor areas exhibit smaller fractions of contralateral preference (SMA-PT, 58\%; PM-PT, 25\%) than M1-PT neurons (88\%) (Tanji et al., 1987). These findings also suggest that rodent M2 is homologous to the primate PM/SMA. Finally, we found that IT neurons had a large fraction of bilateral neurons in both motor areas, especially in M2 (M1-IT, 44\%; M2-IT, 58\%). Together, these data indicate that the M2-IT neurons control contralateral and ipsilateral forelimb movements in a counterbalanced manner, whereas the M1-PT neurons preferentially control contralateral forelimb movements as a final output.

To our knowledge, this is the first study to clearly show distinct motor laterality of forelimb movements in rodent motor cortices, based on the classes and functional activity types of neurons. The combination of neural activity measurement with adequate behavioral experimental systems and genetic engineering techniques in rodents will help to elucidate the network mechanism of motor information processing, including motor control and coordination of individual limbs.

\section{References}

Bruno RM, Simons DJ (2002) Feedforward mechanisms of excitatory and inhibitory cortical receptive fields. J Neurosci 22:10966-10975. Medline

Carvell GE, Simons DJ (1990) Biometric analyses of vibrissal tactile discrimination in the rat. J Neurosci 10:2638-2648. Medline

Catsman-Berrevoets CE, Lemon RN, Verburgh CA, Bentivoglio M, Kuypers HG (1980) Absence of callosal collaterals derived from rat corticospinal neurons. Exp Brain Res 39:433-440. Medline

Chang SW, Snyder LH (2012) The representations of reach endpoints in posterior parietal cortex depend on which hand does the reaching. J Neurophysiol 107:2352-2365. CrossRef Medline

Chang SW, Dickinson AR, Snyder LH (2008) Limb-specific representation for reaching in the posterior parietal cortex. J Neurosci 28:6128-6140. CrossRef Medline

Churchland MM, Yu BM, Ryu SI, Santhanam G, Shenoy KV (2006) Neural variability in premotor cortex provides a signature of motor preparation. J Neurosci 26:3697-3712. CrossRef Medline

Cisek P, Crammond DJ, Kalaska JF (2003) Neural activity in primary motor and dorsal premotor cortex in reaching tasks with the contralateral versus ipsilateral arm. J Neurophysiol 89:922-942. CrossRef Medline

Deffeyes JE, Touvykine B, Quessy S, Dancause N (2015) Interactions between rostral and caudal cortical motor areas in the rat. J Neurophysiol 113:3893-3904. CrossRef Medline

Donoghue JP, Wise SP (1982) The motor cortex of the rat: cytoarchitecture and microstimulation mapping. J Comp Neurol 212:76-88. CrossRef Medline

Ebbesen CL, Doron G, Lenschow C, Brecht M (2017) Vibrissa motor cortex activity suppresses contralateral whisking behavior. Nat Neurosci 20:8289. CrossRef Medline

Evarts EV (1966) Pyramidal tract activity associated with a conditioned hand movement in the monkey. J Neurophysiol 29:1011-1027. Medline

Evarts EV (1973) Motor cortex reflexes associated with learned movement. Science 179:501-503. CrossRef Medline

Gao P, Bermejo R, Zeigler HP (2001) Whisker deafferentation and rodent whisking patterns: behavioral evidence for a central pattern generator. J Neurosci 21:5374-5380. Medline

Georgopoulos AP, Kalaska JF, Caminiti R, Massey JT (1982) On the relations between the direction of two-dimensional arm movements and cell discharge in primate motor cortex. J Neurosci 2:1527-1537. Medline

Griffin DM, Hoffman DS, Strick PL (2015) Corticomotoneuronal cells are "functionally tuned." Science 350:667-670. CrossRef

Harris KD, Shepherd GM (2015) The neocortical circuit: themes and variations. Nat Neurosci 18:170-181. CrossRef Medline

Hazan L, Zugaro M, Buzsáki G (2006) Klusters, NeuroScope, NDManager: a free software suite for neurophysiological data processing and visualization. J Neurosci Methods 155:207-216. CrossRef Medline

Hira R, Terada S, Kondo M, Matsuzaki M (2015) Distinct functional modules for discrete and rhythmic forelimb movements in the mouse motor cortex. J Neurosci 35:13311-13322. CrossRef Medline

Hoshi E, Tanji J (2000) Integration of target and body-part information in the premotor cortex when planning action. Nature 408:466-470. CrossRef Medline

Isomura Y, Harukuni R, Takekawa T, Aizawa H, Fukai T (2009) Microcircuitry coordination of cortical motor information in self-initiation of voluntary movements. Nat Neurosci 12:1586-1593. CrossRef Medline

Isomura Y, Takekawa T, Harukuni R, Handa T, Aizawa H, Takada M, Fukai T (2013) Reward-modulated motor information in identified striatum neurons. J Neurosci 33:10209-10220. CrossRef Medline

Johnston K, DeSouza JF, Everling S (2009) Monkey prefrontal cortical pyramidal and putative interneurons exhibit differential patterns of activity between prosaccade and antisaccade tasks. J Neurosci 29:5516-5524. CrossRef Medline

Kargo WJ, Nitz DA (2003) Early skill learning is expressed through selection and tuning of cortically represented muscle synergies. J Neurosci 23: 11255-11269. Medline

Kargo WJ, Nitz DA (2004) Improvements in the signal-to-noise ratio of motor cortex cells distinguish early versus late phases of motor skill learning. J Neurosci 24:5560-5569. CrossRef Medline

Kawai R, Markman T, Poddar R, Ko R, Fantana AL, Dhawale AK, Kampff AR, Ölveczky BP (2015) Motor cortex is required for learning but not for executing a motor skill. Neuron 86:800-812. CrossRef Medline

Kermadi I, Liu Y, Rouiller EM (2000) Do bimanual motor actions involve the dorsal premotor $(\mathrm{PMd})$, cingulate $(\mathrm{CMA})$ and posterior parietal (PPC) cortices? Comparison with primary and supplementary motor cortical areas. Somatosens Mot Res 17:255-271. CrossRef Medline

Kim H, Ährlund-Richter S, Wang X, Deisseroth K, Carlén M (2016) Prefrontal parvalbumin neurons in control of attention. Cell 164:208-218. CrossRef Medline

Kimura R, Saiki A, Fujiwara-Tsukamoto Y, Ohkubo F, Kitamura K, Matsuzaki M, Sakai Y, Isomura Y (2012) Reinforcing operandum: rapid and 
reliable learning of skilled forelimb movements by head-fixed rodents. J Neurophysiol 108:1781-1792. CrossRef Medline

Kimura R, Saiki A, Fujiwara-Tsukamoto Y, Sakai Y, Isomura Y (2017) Large-scale analysis reveals populational contributions of cortical spike rate and synchrony to behavioural functions. J Neurophysiol 595:385413. CrossRef Medline

Kitsukawa T, Nagata M, Yanagihara D, Tomioka R, Utsumi H, Kubota Y, Yagi T, Graybiel AM, Yamamori T (2011) A novel instrumented multipeg running wheel system, Step-Wheel, for monitoring and controlling complex sequential stepping in mice. J Neurophysiol 106:479-487. CrossRef Medline

Kraskov A, Dancause N, Quallo MM, Shepherd S, Lemon RN (2009) Corticospinal neurons in macaque ventral premotor cortex with mirror properties: a potential mechanism for action suppression? Neuron 64: 922-930. CrossRef Medline

Kurata K (2007) Laterality of movement-related activity reflects transformation of coordinates in ventral premotor cortex and primary motor cortex of monkeys. J Neurophysiol 98:2008-2021. CrossRef Medline

Kurata K (2010) Conditional selection of contralateral and ipsilateral forelimb movements by the dorsal premotor cortex in monkeys. J Neurophysiol 103:262-277. CrossRef Medline

Lambercy O, Schubring-Giese M, Vigaru B, Gassert R, Luft AR, Hosp J (2015) Sub-processes of motor learning revealed by a robotic manipulandum for rodents. Behav Brain Res 278:569-576. CrossRef Medline

Laubach M, Wessberg J, Nicolelis MA (2000) Cortical ensemble activity increasingly predicts behaviour outcomes during learning of a motor task. Nature 405:567-571. CrossRef Medline

Li N, Chen TW, Guo ZV, Gerfen CR, Svoboda K (2015) A motor cortex circuit for motor planning and movement. Nature 519:51-56. CrossRef Medline

Li N, Daie K, Svoboda K, Druckmann S (2016) Robust neuronal dynamics in premotor cortex during motor planning. Nature 532:459-464. CrossRef Medline

Lipski J (1981) Antidromic activation of neurones as an analytic tool in the study of the central nervous system. J Neurosci Methods 4:1-32. CrossRef Medline

Mallet N, Ballion B, Le Moine C, Gonon F (2006) Cortical inputs and GABA interneurons imbalance projection neurons in the striatum of parkinsonian rats. J Neurosci 26:3875-3884. CrossRef Medline

Masamizu Y, Tanaka YR, Tanaka YH, Hira R, Ohkubo F, Kitamura K, Isomura Y, Okada T, Matsuzaki M (2014) Two distinct layer-specific dynamics of cortical ensembles during learning of a motor task. Nat Neurosci 17:987-994. CrossRef Medline

Morishima M, Kawaguchi Y (2006) Recurrent connection patterns of corticostriatal pyramidal cells in frontal cortex. J Neurosci 26:4394-4405. CrossRef Medline

Mushiake H, Inase M, Tanji J (1991) Neuronal activity in the primate premotor, supplementary, and precentral motor cortex during visually guided and internally determined sequential movements. J Neurophysiol 66:705-718. Medline

Nakayama Y, Yokoyama O, Hoshi E (2015) Distinct neuronal organizations of the caudal cingulate motor area and supplementary motor area in monkeys for ipsilateral and contralateral hand movements. J Neurophysiol 113:2845-2858. CrossRef Medline

Narayanan NS, Laubach M (2009) Delay activity in rodent frontal cortex during a simple reaction time task. J Neurophysiol 101:2859-2871. CrossRef Medline

Neafsey EJ, Bold EL, Haas G, Hurley-Gius KM, Quirk G, Sievert CF, Terreberry RR (1986) The organization of the rat motor cortex: a microstimulation mapping study. Brain Res 11:77-96. CrossRef Medline

Nonomura S, Fujiwara-Tsukamoto Y, Kajihara T, Fujiyama F, Isomura Y (2017) Continuous membrane potential fluctuations in motor cortex and striatum neurons during voluntary forelimb movements and pauses. Neurosci Res 120:53-59. CrossRef Medline
Panigrahi B, Martin KA, Li Y, Graves AR, Vollmer A, Olson L, Mensh BD, Karpova AY, Dudman JT (2015) Dopamine is required for the neural representation and control of movement vigor. Cell 162:1418-1430. CrossRef Medline

Peters AJ, Chen SX, Komiyama T (2014) Emergence of reproducible spatiotemporal activity during motor learning. Nature 510:263-267. CrossRef Medline

Reep RL, Corwin JV, Hashimoto A, Watson RT (1987) Efferent connections of the rostral portion of medial agranular cortex in rats. Brain Res Bull 19:203-221. CrossRef Medline

Reiner A, Jiao Y, Del Mar N, Laverghetta AV, Lei WL (2003) Differential morphology of pyramidal tract-type and intratelencephalically projectingtype corticostriatal neurons and their intrastriatal terminals in rats. J Comp Neurol 457:420-440. CrossRef Medline

Rouiller EM, Moret V, Liang F (1993) Comparison of the connectional properties of the two forelimb areas of the rat sensorimotor cortex: support for the presence of a premotor or supplementary motor cortical area. Somatosens Mot Res 10:269-289. CrossRef Medline

Saiki A, Kimura R, Samura T, Fujiwara-Tsukamoto Y, Sakai Y, Isomura Y (2014) Different modulation of common motor information in rat primary and secondary motor cortices. PLoS One 9:e98662. CrossRef Medline

Saiki A, Sakai Y, Fukabori R, Soma S, Yoshida J, Kawabata M, Yawo H, Kobayashi K, Kimura M, Isomura Y (2017) In vivo spiking dynamics of intra-and extratelencephalic projection neurons in rat motor cortex. Cereb Cortex. Advance online publication. Retrieved Jan. 30, 2017. doi: 10.1093/cercor/bhx012. CrossRef Medline

Shenoy KV, Sahani M, Churchland MM (2013) Cortical control of arm movements: a dynamical systems perspective. Annu Rev Neurosci 36: 337-359. CrossRef Medline

Shepherd GM (2013) Corticostriatal connectivity and its role in disease. Nat Rev Neurosci 14:278-291. CrossRef Medline

Shima K, Mushiake H, Saito N, Tanji J (1996) Role for cells in the presupplementary motor area in updating motor plans. Proc Natl Acad Sci U S A 93:8694-8698. CrossRef Medline

Simons DJ (1978) Response properties of vibrissa units in rat SI somatosensory neocortex. J Neurophysiol 41:798-820. Medline

Soma S, Suematsu N, Shimegi S (2014) Efficient training protocol for rapid learning of the two-alternative forced-choice visual stimulus detection task. Physiol Rep 2:e12060. CrossRef Medline

Takekawa T, Isomura Y, Fukai T (2010) Accurate spike sorting for multiunit recordings. Eur J Neurosci 31:263-272. CrossRef Medline

Takekawa T, Isomura Y, Fukai T (2012) Spike sorting of heterogeneous neuron types by multimodality-weighted PCA and explicit robust variational Bayes. Front Neuroinform 6:5. CrossRef Medline

Tanji J, Okano K, Sato KC (1987) Relation of neurons in the nonprimary motor cortex to bilateral hand movement. Nature 327:618-620. CrossRef Medline

Tanji J, Okano K, Sato KC (1988) Neuronal activity in cortical motor areas related to ipsilateral, contralateral, and bilateral digit movements of the monkey. J Neurophysiol 60:325-343. Medline

Tantirigama ML, Oswald MJ, Clare AJ, Wicky HE, Day RC, Hughes SM, Empson RM (2016) Fezf2 expression in layer 5 projection neurons of mature mouse motor cortex. J Comp Neurol 524:829-845. CrossRef Medline

Tomita H, Sugano E, Fukazawa Y, Isago H, Sugiyama Y, Hiroi T, Ishizuka T, Mushiake H, Kato M, Hirabayashi M, Shigemoto R, Yawo H, Tamai M (2009) Visual properties of transgenic rats harboring the channelrhodopsin-2 gene regulated by the thy-1.2 promoter. PLoS One 4:e7679. CrossRef Medline

Weinrich M, Wise SP (1982) The premotor cortex of the monkey. J Neurosci 2:1329-1345. Medline

Yttri EA, Dudman JT (2016) Opponent and bidirectional control of movement velocity in the basal ganglia. Nature 533:402-406. CrossRef Medline 